\title{
HOW TO BE A SUCCESSFUL ORGANIC CHEMIST
}

Dr. Alexander Sandtorv, Ph. D.

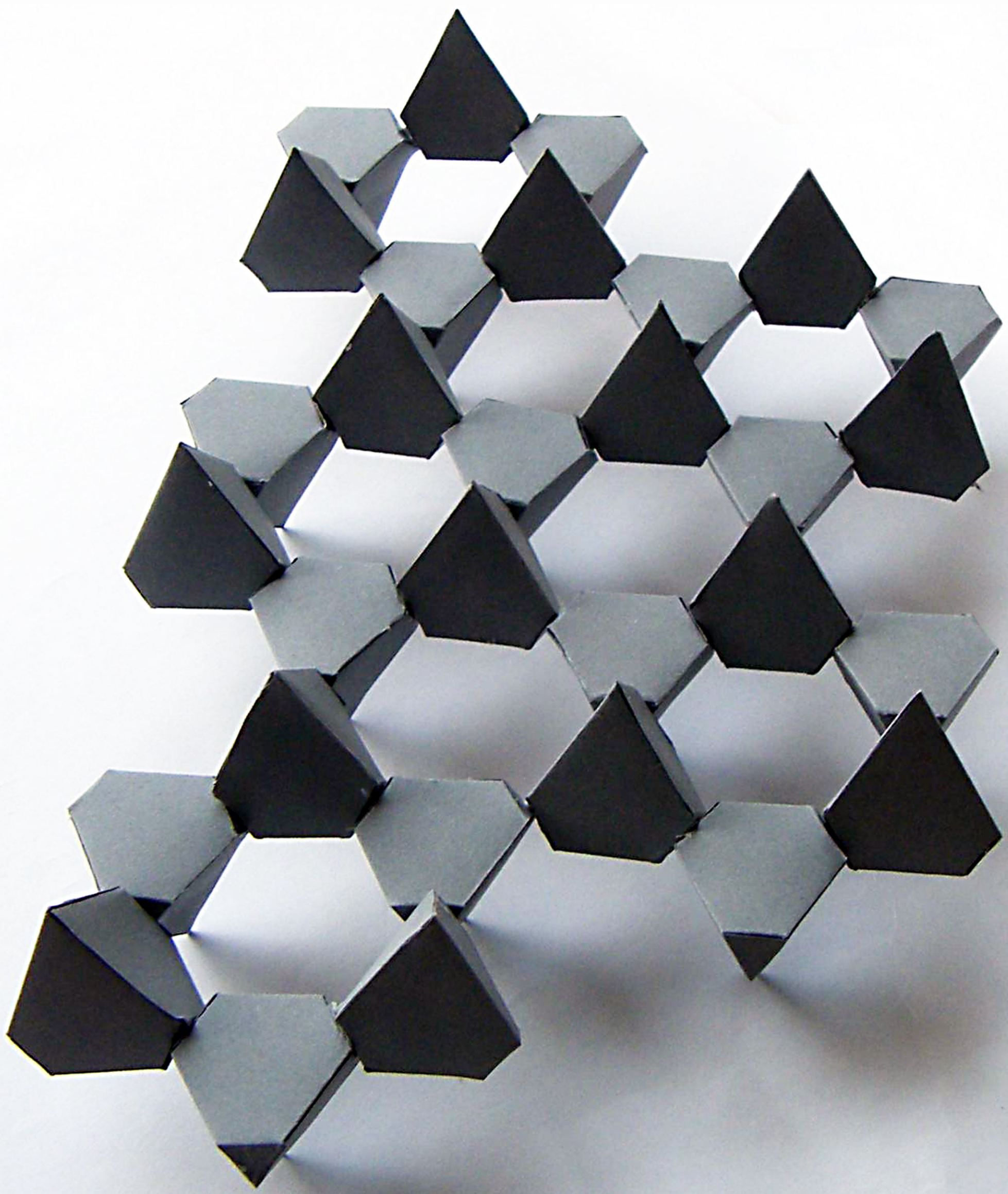




\section{Accessibility Statement}

PDXScholar supports the creation, use, and remixing of open educational resources (OER). Portland State University (PSU) Library acknowledges that many open educational resources are not created with accessibility in mind, which creates barriers to teaching and learning.

PDXScholar is actively committed to increasing the accessibility and usability of the works we produce and/or host. We welcome feedback about accessibility issues our users encounter so that we can work to mitigate them. Please email us with your questions and comments at pdxscholar@pdx.edu.

\section{Accessibility of How to be a Successful Organic Chemist}

This book contains the following accessibility and usability features:

\section{File Formats Available}

- This book is only available in PDF format; an editable (Microsoft Word) version of this book is not available.

Known Issues/Potential barriers to accessibility

\section{Organization of content}

- The content is organized under headings and subheadings, but it does not include a Table of contents.

\section{Images}

- Not all images include alternative text

- Images rely on color to convey meaning

\section{Font Size and formatting}

- Font size is not 12 point or higher for body text

- The book contains repeated characters or excessive spaces

\section{Links}

- Not all hyperlinks use descriptive link text.

\section{Lists and Tagging}

- The book contains tag issues and the content may not be correctly or consistently tagged.

If you have trouble accessing this material, please contact us at pdxscholar@pdx.edu. 
This accessibility statement has been adopted and adapted from Accessibility Statement and Appendix A: Checklist for Accessibility found in Accessibility Toolkit - 2nd Edition by BCcampus, and is licensed under a_Creative Commons Attribution 4.0 International License. 
(C) 2018 Alexander H. Sandtorv

Originally published in 2017

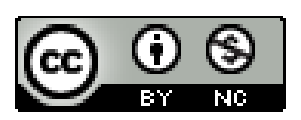

This work is licensed under a

Creative Commons Attribution-NonCommercial 4.0 International License

You are free to:

- Share - copy and redistribute the material in any medium or format

- Adapt - remix, transform, and build upon the material

The licensor cannot revoke these freedoms as long as you follow the license terms.

Under the following terms:

- Attribution - You must give appropriate credit, provide a link to the license, and indicate if changes were made. You may do so in any reasonable manner, but not in any way that suggests the licensor endorses you or your use.

- NonCommercial - You may not use the material for commercial purposes.

This publication was made possible by PDXOpen publishing initiative

Published by Portland State University Library

Portland, OR 97207-1151

Cover design by Ryan Brewer 


\section{INTRODUCTION}

I am a firm believer that organic chemistry can save the world. Or at least, make it better. Within the realm of organic chemistry is endless opportunity for innovation that has undoubtedly made its mark on our civilization.

It is my pleasure to introduce you to practical organic chemistry here at PSU. The following text will outline key features of the labs and my hope is that it will help your lab experience be as fulfilling as possible.

Speaking from the experience, I have seen many students struggle through the organic chemistry labs. My primary motivation in writing this text, was to make sure all our students had the answers to the questions nobody really asked (or were too afraid to ask). Why did my recrystallization not give me any crystals? How do I analyze my data? How do I find the necessary safety data on the chemicals I will be working with?

The text is divided into three parts. Preparation, execution and analysis.

Chapter $1-3$ is all about the preparation. We will cover how to prepare for the labs, all the important safety features of working in a lab environment, and the most important laboratory techiques encountered.

Chapter 4 is about the execution of the experiment. We will investigate why experiments fail, and how to reach a deeper understanding in the labs.

Chapter 5 and 6 is all about the analysis. We will look at data, how to interpret data, and how to put it all together in a logical and scientifically sound way.

I am thankful the Portland State University Library for generously funding this project, and I especially want to thank Karen Bjork for all her excellent technical assistance. I am especially proud to be part of a project that is open access, to ensure that information is available to all, free of charge.

I want to thank my four student readers, Carlee Moser, Monica Joseph, Daniel Gaynor, and Antonio Montano. The student perspective was very much appreciated. I would like to thank Sarah Bartlett for amazing assistance with the preparation of the manuscript. I would also like to thank my excellent peer reviewers Prof. Alan Shusterman (Reed College) and Prof. Louis Kuo (Lewis and Clark College). Their thoughts and comments lifted the manuscript immensely. Lastly I would like to thank Ryan Brewer for his assistance with the type setting of the manuscript.

Dr. Alexander H. Sandtorv, August 2017 


\section{HOW TO PREPARE FOR AN ORGANIC CHEMISTRY EXPERIMENT}

\section{INTRODUCTION}

Laboratory courses can be magical. They can be enlightening experiences that open your eye to a big picture. A laboratory experience should work in tandem with a lecture course, and fully realize concepts, techniques and reactions that you have heard of. Unfortunately, they can also be discouraging experiences. One of the key factors that dictates which experience you will have, is preparation.

A rewarding aspect of a well-prepared experiment is that it can firmly cement the information that you have obtained through studying in a way that is far superior to simply reading about it. Your knowledge evolves beyond routine memorizing to real understanding, because you have seen the reaction and principles with your own eyes. The synergy between a lecture course and a lab component should not be underestimated.

In this chapter I outline a systematic way of preparing for any organic chemistry experiment to ensure that you succeed in the laboratory and that you leave with an optimal experience. Seeing, after all, is believing. I discuss how to make and use the flow diagrams, how to obtain relevant safety information about the chemicals you are handling, and how to use your note-book to prepare efficiently.

\subsection{READING, DRAWING AND UNDERSTANDING; THE POWER OF VISUALIZATION}

There are several reasons why a systematic plan for preparation is so integral to a laboratory experience, but it boils down to the fact that an organic chemist must have information that far supersedes the information provided in a typical lab manual. When you are performing an experiment, there is little time to look up densities, perform calculations, look up safety and hazard information for chemicals and do any in-depth research on the theory behind the techniques you use. You must bring all this information with you. In many cases the lack of this information can have detrimental consequences for your experiment, your learning outcome and last but not least the safety of the laboratory.

So how can you prepare better?

Let us start by looking at the procedure. It is a recipe that describes the order of events, and the key operations that must be performed. However, just reading the procedure will never prepare you adequately. ${ }^{1}$ Why is that? The first reason, is that there is far too much material to remember, at any given time, and events that happen early in the experiment, will influence the outcome later. The second reason, is that any lab manual, even the ones designed for undergraduate use, never spells out everything that must be done. A third reason, is that any lab manual expects that the reader has some expertise and some level of experience.

This is something you most likely already know, from your everyday life.

Let's say that you want to follow a recipe you are not familiar with to make a birthday cake. You start on step 1 and work your way through the recipe, but after a while, you run into a problem.

Every term we see many students that simply read the procedure (sometimes repeatedly), and end up confused and suffering through a sub-par experience. 
The recipe calls for four cups of flour, but you only have two. You have a hot oven that is ready for the cake batter, a half-made frosting, and a batter that is not ready at all. You switch off the oven and rush to the store for more flour. By the time you come home, the oven is cold (and must be re-heated), and the frosting has split because you left it out in room temperature for half an hour.

This example might seem far removed from the lab, but it is perfectly transferrable. Because you had not read the recipe (or prepared adequately), you wasted time and part of your cake. Nobody wants to waste a cake.

If we take another cake-analogy and move closer to what an experiment in a lab might look like, we can say that you have a recipe for your great grandmother's favorite chocolate cake that you wish to make. Here is the start of the recipe:

\section{GREAT GRANDMOTHER ELSA'S FAVORITE CHOCOLATE CAKE:}

1. Pre-heat oven.

2. Beat 1 stick of butter with 1 cup of sugar until mixture is light.

3. Add 3 eggs, one at the time, to the sugar and butter mixture.

4. In a bowl, add 2 cups of flour, 1 small spoon of baking powder and 1 cup of cacao-powder.

Let us think of this recipe as a lab procedure. What we absolutely do not want is to read the procedure once, and then go to the kitchen (lab) and start to make the cake (the experiment). Why not? Well, we will immediately run into many problems. In step one; Elsa says to pre-heat the oven. To what temperature, exactly? Moreover, in step 2, we beat together butter and sugar. With what? And in what? What size bowl do we need? What will we use to do the actual beating? In step 3, we add one egg at the time, but surely, we should carefully beat in each egg, before the next one is added? Elsa does not clarify that, but if we did not have to beat in each egg, there would be no point in adding one egg at the time.

As in Elsa's recipe and in a lab manual (even one designed for undergraduate use) we absolutely need to visualize the recipe or procedure, before we move on. You might have noted that through the act of visualizing Elsa's recipe, I identified several issues and questions. We want to be aware of these up front, so that we have the answers and solutions before we start cooking.

Visualization is the act of seeing the procedure before your eyes, as you read it. This is a very important part of preparation, as it allows you to identify any problems, questions or issues before you go into the lab. It also allows you time to look up valuable information. Elsa did not give us the exact temperature for baking her cake, but we could always look up what baking times for similar cakes are.

At this stage, I want to introduce another powerful tool: the flow chart. The flow chart is a graphical overview of a procedure that should contain the essentials needed to complete the procedure. The flow chart is a product created after visualization, and it should be something you bring with you to lab. It should outline the flow of the experiment, but also provide something additional to the procedure: namely, the answers to all those questions that came up during visualization.

In the figure shown I have shown a flow chart for Great Grandmother Elsa's recipe. Note that I have included all the information from the questions above.

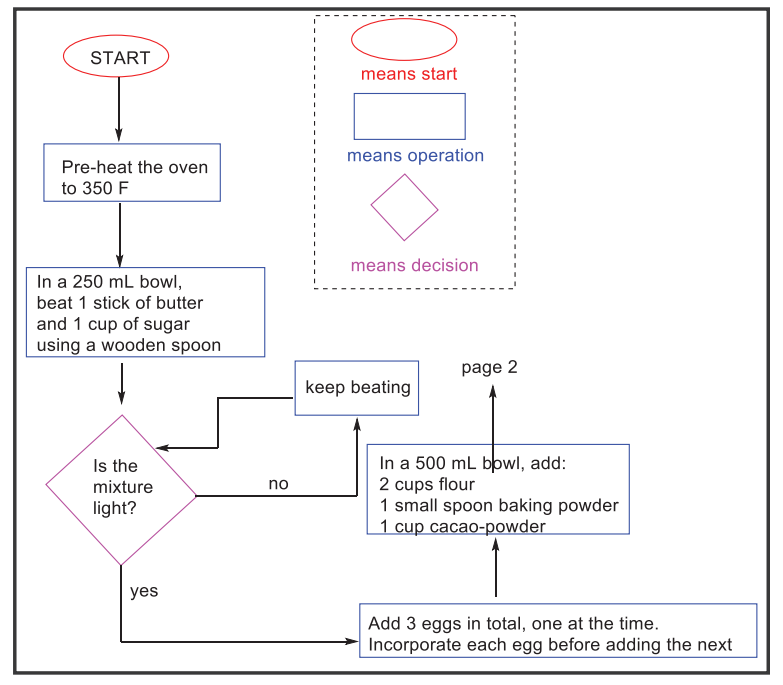

Figure 1.1. An example of a flow chart for Great Grandmother Elsa's chocolate cake. Note that I have incorporated the questions that arose during the visualization phase. 
I have the temperature of the oven (based on a search I did for similar cakes), I have the sizes of the bowl, and the added clarification to beat in each egg, before adding the next.

\subsection{SAFETY IS KEY: HOW TO FIND RELEVANT SAFETY INFORMATION AND READ SAFETY DATA SHEETS}

Safety is a number one concern for everyone working in a lab. Safety concerns have many different manifestations, and in general, we always work to make everyone as safe as possible by creating a standard procedure for whenever is in the lab. In any given situation in lab (and there are no exceptions), we use the following:

1. Lab goggles. Our eyes are very delicate, and almost all chemicals are detrimental for our eye health. Goggles are worn at all times, and they are never removed in the lab. Goggles must be splash proof, which means that they protect the eyes from multiple angles, should chemicals splash.

2. Lab coat. The coat is the first line of defense against chemicals that may get in contact with us, and the coat also provides a first line of defense for our clothes.

3. Fume-hoods. A fume-hood is a very important part of the lab environment. It is a hood equipped with an adjustable sash, where the air flow inside the hood is very high. This removes any vapors quickly and safely. Everything that you do in an organic chemistry lab, is done in a fume-hood to minimize your exposure to fumes.

In many situations, the use of gloves is also appropriate. This is covered in detail in chapter 3.

The scary reality of working in a lab is that chemicals are dangerous. All substances have some inherent dangers associated with them, and although we spend our times exposed to many chemicals outside of the lab, the lab is a place where we can expose ourselves to a greater variety of hazardous chemicals. As a base line, we will treat any chemical as dangerous, and the utmost of care should always be taken to ensure that exposure is at a minimum. Because chemicals do vary in terms of their properties and hazards, it is also necessary to know the exact hazards of each chemical.

Where should you go to look up, identify and familiarize yourself with this information?

The answer is Safety Data Sheets (SDS).

SDS are legally required information sheets that provide an overview of the safety and hazards associated with a given chemical or mixture. There may be thousands of data sheets available for any given chemical, so before I move on, I would recommend using Sigma Aldrich as the main supplier of relevant and reliable data sheets. ${ }^{2}$

The SDS are compiled by the Globally Harmonized System of classification and labeling of chemicals (GHS), an internationally recognized system that standardizes safety and hazard information for chemicals. That means that people from all over the world should be able to access any given SDS and find the same types of information, presented in the same way.

Knowing how to work with SDS is essential for anyone working in a laboratory environment, because they contain information on the risks, hazards and safety issues associated with the chemicals in use. SDS give an informed backdrop to the experiment and helps you make safe and good choices when handling chemicals.

To contextualize this, just imagine that you were planning to handle a chemical that is explosive in contact with water. Knowing this hazard in advance is obviously key to your safety. On the other hand, what if you were planning to handle a carcinogenic substance (a substance that causes cancer)? You would obviously want to know these risks beforehand so that you could work in the safest way possible. Furthermore, if you ever plan to work in a professional laboratory, consulting SDS will be a very important part of your preparation.

The site http://sigmaaldrich.com/ is usually a good starting point. You can search for chemicals by name or structure. 
Let us have a closer look at how a SDS is formatted.

The SDS for any given compound is typically organized into several categories, such as hazards, toxicity, composition, and first aid measures, to name a few.

The information found in a SDS can be misleading if misinterpreted. As an example, let us examine the SDS of sodium chloride $(\mathrm{NaCl})$, which is table salt. Under category 4, which is first-aid information, the SDS states that if the substance is in contact with skin to "Wash off with soap and plenty of water. Consult a physician." That seems ridiculous. I doubt that any of us call a physician if we get salt on our fingers while we are eating fries, but the restaurant cook who is dipping their fingers into a bowl of salt eight hours a day might wonder if it will cause any harmful effects. The SDS also lists information about the "acute toxicity" of sodium chloride. The data stated is $\mathrm{LD}_{50}$ Oral Rat $3,550 \mathrm{mg} / \mathrm{kg}$. How does one interpret this?

$\mathrm{LD}_{50}$ Oral Rat refers to the amount of salt that will kill $50 \%$ of the rats who swallow it. Because rats come in all sizes, the amount is expressed as ratio between mass of compound and mass of rate. In this case, 3550 $\mathrm{mg}$ (or $3.55 \mathrm{~g}$ ) of sodium chloride will kill $50 \%$ of rats who weigh $1 \mathrm{~kg}$.

If we assume that human bodies work like rats, we can find a lethal dose for half a population of humans. If we assume that a human weigh $100 \mathrm{lb}$ (which is a convenient number) or $45.4 \mathrm{~kg}$, then 45.4 x $3.55=161$ $\mathrm{g} \mathrm{NaCl}$ might be lethal to $50 \%$ of the humans who swallow it. $161 \mathrm{~g}$ is over $1 / 3 \mathrm{lb}$ of salt, so the fries would probably kill you before the salt did, but there is how you interpret this figure.

As you can see from this example, the reliable information in the SDS has to be worked out ina realistic context. In this particular case, we have learned that sodium chloride can be lethal if ingested in (relatively) large amounts.

Here are some of the hazards categories that SDS frequently identify for organic chemicals, and some notes about each category to help you get started. Please note that this list is not exhaustive.

Table 1 . Some common safety categories

\begin{tabular}{|c|c|c|c|}
\hline Category & Type & What does it mean? & Additional comments. \\
\hline Flammable & Physical & $\begin{array}{l}\text { A liquid, gas, solid or solution } \\
\text { is flammable. Usually a spark or }\end{array}$ & $\begin{array}{l}\text { Most organic liquids are flammable, so the risk of starting a fire is always } \\
\text { present and necessary steps must always be taken to avoid such risks. }\end{array}$ \\
\hline \multirow[t]{2}{*}{ Oxidizing } & Physical & $\begin{array}{l}\text { The compound is a strong } \\
\text { oxidant. }\end{array}$ & $\begin{array}{l}\text { If you mix an oxidant with a reductant, usually a strongly exothermic reaction } \\
\text { happens. Care should be taken to avoid this. }\end{array}$ \\
\hline & & & Some oxidizers must also be disposed of in a particular way. \\
\hline Pyrophoric & Physical & $\begin{array}{l}\text { The compound ignites } \\
\text { spontaneously in contact with air. }\end{array}$ & $\begin{array}{l}\text { Pyrophoric substances are very dangerous, and usually require handling in } \\
\text { inert gas. }\end{array}$ \\
\hline Toxicity & Health & $\begin{array}{l}\text { A substance is toxic. This category } \\
\text { is vast, and requires specific }\end{array}$ & $\begin{array}{l}\text { Most organic molecules are toxic by numerous modes. We have dedicated a } \\
\text { separate section to toxicity below this table. }\end{array}$ \\
\hline Skin irritation & Health & $\begin{array}{l}\text { A substance irritates the skin and/ } \\
\text { or mucus membranes }\end{array}$ & $\begin{array}{l}\text { Most organic chemicals irritate the skin. If the compounds are solids, gloves } \\
\text { often offer sufficient protection, assuming the substances do not permeate }\end{array}$ \\
\hline $\begin{array}{l}\text { Eye irritation } \\
\text { /damage }\end{array}$ & Health & $\begin{array}{l}\text { A substance irritates or } \\
\text { permanently damages the eye }\end{array}$ & $\begin{array}{l}\text { Because the eye is so delicate, most chemicals and substances damage the eye. } \\
\text { One of the most basic safety feature of any lab, is that the people working }\end{array}$ \\
\hline Carcinogen & Health & $\begin{array}{l}\text { A substance that is suspected } \\
\text { or known to cause cancer in } \\
\text { mammalian cells. }\end{array}$ & $\begin{array}{l}\text { This is a very important category, as substances that are either suspected or } \\
\text { known carcinogens must be handled with extreme care. It is important to } \\
\text { note the two subcategories of suspected and known. In practical lab work, } \\
\text { you should deal with both these chemicals the same way: very carefully }\end{array}$ \\
\hline $\begin{array}{l}\text { Reproductive } \\
\text { toxicity }\end{array}$ & Health & $\begin{array}{l}\text { The substance damages the } \\
\text { reproductive system }\end{array}$ & $\begin{array}{l}\text { This is a very important category, as chemicals that damages the reproductive } \\
\text { system can have very detrimental effects. }\end{array}$ \\
\hline
\end{tabular}


How do you use the SDS information in your preparation phase?

1. Read all SDS for all chemicals encountered in the experiment. Pay particular attention to category 2, which contains a summary of the hazard statements for the chemical.

2. Analyze the information in the SDS. Think carefully about what measures can (and should!) be taken to avoid exposure, and to minimize the potential risks associated with the chemical.

3. Write it down. Include the major hazard statements for each chemical in your notebook. Also, include any important analysis you have done.

\subsection{A SPECIFIC SDS}

Let us use all the information we have accumulated in the last sub-chapter, and use a real case example to find the relevant information we need. We will analyze the SDS of toluene, as a specific example.

We will focus on category 2 in the SDS, which is a good starting point for most SDS. This category contains hazard information. Category 2.1 contains the most important summary of the hazards and safety information associated for that chemical. For toluene, we have the following seven items:

1. Flammable liquids (Category 2), H225

2. Skin irritation (Category 2), H315

3. Reproductive toxicity (Category 2), H361

4. Specific target organ toxicity -single exposure (Category 3), Central nervous system, H336

5. Specific target organ toxicity -repeated exposure (Category 2), H373

6. Aspiration hazard (Category 1), H304

7. Acute aquatic toxicity (Category 2 ), $\mathrm{H} 401$

Each of these items should go in the lab notebook, if toluene is used for that experiment. As a deeper analysis, we will also go through each of these seven items, and evaluate the relative hazard. As outlined in the previous sub-chapter, our aim is to analyze each item and think carefully about measures that we should take to avoid exposure and risk.

Items 1 and 2 (flammable liquid and skin irritation) are, as we have seen, typical for most organic chemicals and we follow good laboratory hygiene and work in a well ventilated fume-hood. We also wear goggles and a lab coat at all times. Item three, which deals with reproductive toxicity, ${ }^{3}$ is worth making a note of. That is an effect that we absolutely should be aware of when dealing with the chemical. Items 4 and 5 are also noteworthy. These two items say that the chemical has target toxicity both for single and repeated exposure. The first is more serious than the latter, because only one exposure of the chemical can have central nervous system toxicity. We therefore want to do everything we can to avoid contact with the chemicals. A further analysis shows that nitrile gloves offer fair protection, so we want to make sure to wear these gloves whenever handling toluene. ${ }^{4}$ Item 6 is common for most organic chemicals, as inhalation of most organic chemicals is damaging. Working in the fume-hood is one measure to limit inhalation. Item 7 is worth noting as well, and following proper chemical handling, ${ }^{5}$ we make sure to never pour chemicals down the drain.

The above analysis might be deeper than you are used to, but it is very important for your long-term health and well-being. One of the main goals of a chemistry lab course is to learn about safety in that environment.

Reproductive toxicity means a substance that in some way interfers with reproduction. It includes effects on sexual function, and fertility in both males and females, as well as developmental toxicity in the offspring.

Chapter 3 covers the use of gloves

Chapter 3 covers safe handling of chemicals 
In conclusion, several safety-related issues are important for preparation.

1. Find and read SDS for all chemicals used

2. Find the main safety and hazard statements for each chemical used

3. Include the main safety and hazard statements in your note book

\subsection{THE NOTEBOOK - MAKING SURE THE NUMBERS ADD UP}

At PSU, it is required to use and keep a notebook. This notebook will be used both in the preparation of the experiment, and during the experiment itself. We will focus on how to use the notebook in the preparation phase of an experiment. In chapter 4.4 we will discuss the use of the notebook during the experiment.

When stepping into the lab, your notebook should contain the following:

1. A title and a balanced reaction scheme, if a synthesis is planned. If not, the relevant chemical structures of the compounds of interest should be shown.

2. A flow-diagram that outlines the experiment, where you have included the most relevant information.

3. A table or overview that shows all chemicals handled, and their risk and hazard statements.

4. A synthesis table that shows the quantities of the reagents/reactants that you plan to use, with room to fill in the quantities you actually used.

5. A table that shows the properties of the reagents/reactants and reaction solvents used in the procedure.

The synthesis table should contain the most important physical properties of the reactant(s), reagent(s) and product(s). This table should contain the quantities you should measure, with room to add your actual measurements. This format allows you to order all the necessary measurements in the same place. An example is shown in table 2 . There are many examples where this information is important in lab. If you for example measure $0.4 \mathrm{~mL}$ of benzaldehyde, instead of the $0.3 \mathrm{~mL}$ the procedure called for, you need to scale the procedure up. That means that the amount of $\mathrm{NaBH}_{4}$ also must be changed. For this process, you need the density of benzaldehyde, the molecular weight of benzaldehyde to find the moles, and the molecular weight of $\mathrm{NaBH}_{4}$.

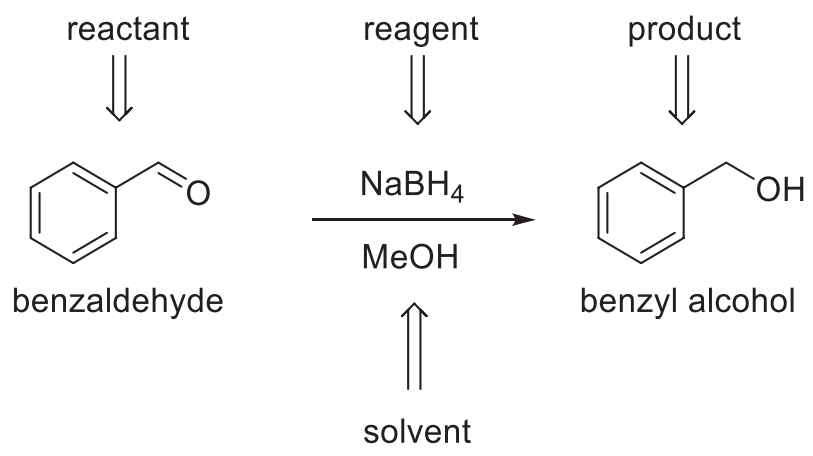

Scheme 1. A reaction scheme in the note book

Table 2. Example of a synthesis table for reagent(s), reactant(s) and product(s)

\begin{tabular}{|c|c|c|c|c|c|c|}
\hline Compound & $\begin{array}{l}\text { Mw } \\
{[\mathrm{g} / \mathrm{mol}]}\end{array}$ & $\begin{array}{l}\mathrm{d} \\
{[\mathrm{g} / \mathrm{mL}]}\end{array}$ & $\begin{array}{l}\mathrm{V} \\
{[\mathrm{mL}]}\end{array}$ & $\begin{array}{l}\text { m } \\
{[\mathrm{g}]}\end{array}$ & $\begin{array}{l}\text { moles } \\
{[\mathrm{mmol}]}\end{array}$ & $\begin{array}{l}\text { Yield } \\
{[\%]}\end{array}$ \\
\hline Benzaldehyde & 106.7 & 1.044 & 0.30 & 0.31 & 2.9 & - \\
\hline $\mathrm{NaBH}_{4}$ & 37.8 & - & - & 0.15 & 3.9 & - \\
\hline Benzyl alcohol & 108.1 & 1.044 & - & $0.21^{*}$ & $1.9^{*}$ & $65.6^{*}$ \\
\hline
\end{tabular}

*The values for benzyl alcohol is based on the procedure, that states that a yield of $65.6 \%$ is expected. 
The table for physical properties is used not only for the reagent(s), reactant(s) and product(s), but also reaction solvent, work-up chemicals and others. This table is very handy in the lab, as the physical properties of the chemicals play a large part in the work-up of any reaction.

Table 3. Example of a table for physical data

\begin{tabular}{llllll}
\hline Compound & State & $\begin{array}{l}\text { Boiling } \\
\text { point }\end{array}$ & $\begin{array}{l}\mathbf{d} \\
{[\mathrm{g} / \mathrm{mL}]}\end{array}$ & $\begin{array}{l}\text { Solubility in } \\
\text { water } \\
{\left[{ }^{\circ} \mathrm{C}\right]}\end{array}$ & $\begin{array}{l}\text { Solubility in } \\
\text { diethyl ether }\end{array}$ \\
\hline Benzaldehyde & Liquid & 178 & 1.044 & 0.3 & \\
$\mathrm{NaBH}_{4}$ & Solid & - & Not relevant & Decomposes & Non soluble \\
Benzyl alcohol & Liquid & 205 & 1.044 & 3.50 & Soluble \\
Diethyl ether & Liquid & 36 & 0.71 & 6.05 & - \\
Methanol & Liquid & 65 & 0.79 & Soluble & Soluble \\
& & & & & \\
\hline
\end{tabular}




\section{INTRODUCTION}

Organic chemistry is an art-form and a craft. In research, we often talk about candidates that "have it", the "magic touch" to get a compound analytically pure, or solve a very difficult synthetic challenge. In this chapter we will go through some common techniques that you will encounter in the organic chemistry labs. The aim of this chapter is not to give you a comprehensive introduction in the theory behind these techniques, but to highlight technical aspects to make sure you also develop this "magic touch."We will discuss recrystallization, distillation, liquid-liquid extraction, TLC, chromatography and lastly sublimation.

\subsection{RECRYSTALLIZATION}

Recrystallization is a laboratory technique for purifying solids. The key features of this technique is causing a solid to go into solution, and then gradually allowing the dissolved solid to crystallize. Sounds easy, doesn't it? It is actually a very challenging process to get completely right. The goal, is to obtain a compound in high purity as uniform crystals. Recrystallization is therefore a purification technique.

The key features necessary for a successful recrystallization process, are a very controlled temperature decrease and sufficient time. Because most solids have a better solubility at higher temperatures, we can saturate or almost saturate a solution at high temperature (usually the boiling temperature of the solvent), and then slowly allow the solution to reach room temperature. As the temperature starts to decrease, so does the solubility of the compound. As the solubility decreases, the solution at some point becomes supersaturated and crystals will start to form.

Before we move on, let me address the main problem associated with crystallization: the formation of precipitate, versus crystals. A precipitate is simply a mixture of compounds in the solution that crash out. This can happen for a variety of reasons, but a student may have taken the very hot solution and placed it directly on a cold surface to cool (a process called "shock cooling") or even plunged the hot solution into an ice bath. A precipitate may not pure, because it can contain several compounds. Crystals, however, are often composed solely of one compound. It is very easy to get a precipitate, but very difficult to get crystals.

We already mentioned temperature and time being key factors for successful recrystallization. Precipitation typically occurs when the temperature has not been lowered gradually. Furthermore, we must not disturb the solution (or its container) as it cools (even though it is tempting). Disturbing the solution can break up any seed crystals ${ }^{6}$ that have started growing. ${ }^{7}$ 


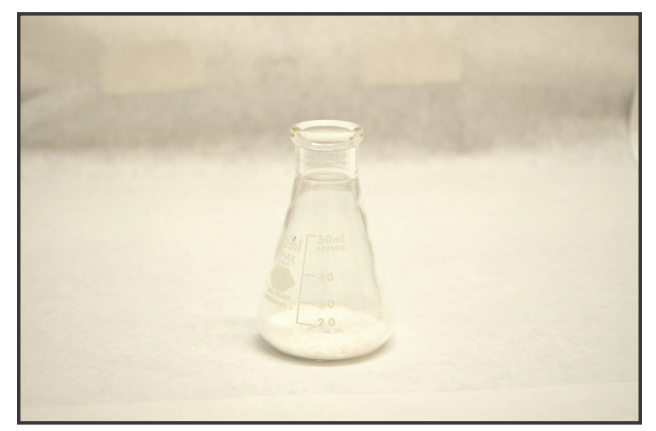

(A) The crude material is transferred to a suitable crystallization vessel.

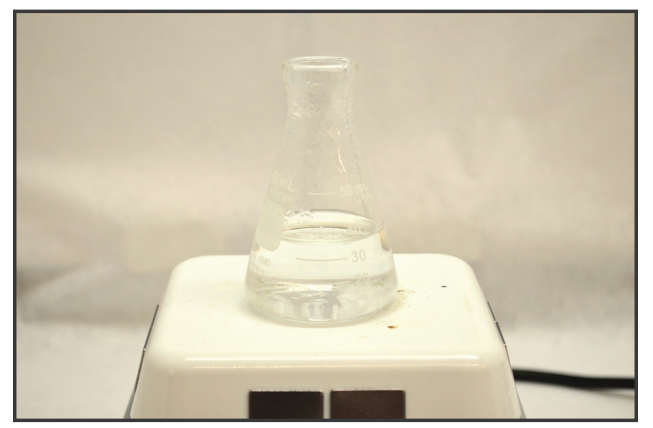

(B) The crude material is dissolved in a solvent, and gently heated.

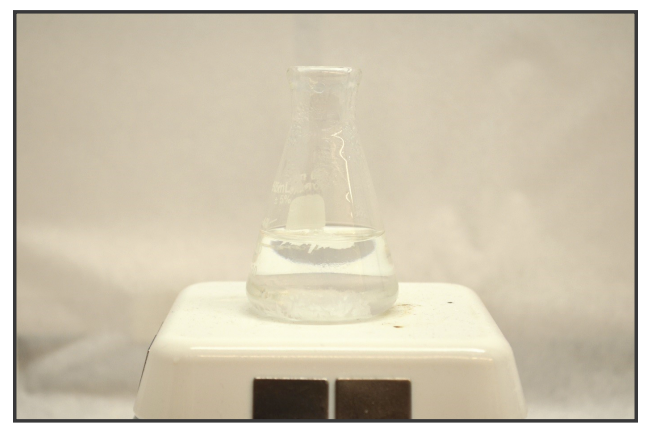

(C) The solution is allowed to gently and slowly cool down. Notice the crystals growing in the solution.

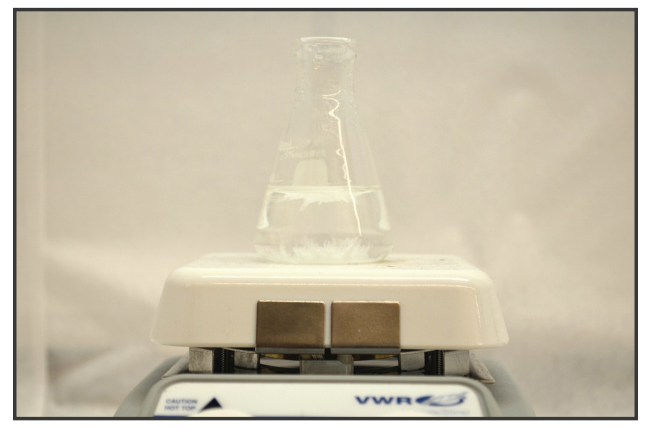

(D) The solution is cooled to room temperature, leading to the formation of large crystals.

Figure 2.1. Key steps in a recrystallization process
Please also note that some compounds simpl crystallize more easily than others. More rigid molecules are, as a rule, easier to crystalize. ${ }^{8}$ Rigid, in this context, mean compounds that contain fewer bond capable of undergoing internal rotation, so that there are fewer possible conformers possible.

Let us go through a recrystallization process, focusing on technical aspects and trouble shooting.

How to perform a recrystallization:

1. The crude impure solid is dissolved in hot solvent. If some solid remains undissolved after adding solvent, it is likely to be an impurity and should be removed by filtrering the (hot!) solution.

Typical problems: Adding too much solvent so that the product does not crystallize later. Filtering the hot solution too slowly so that the solvent cools and the solid starts crystallizing in the funnel and/or on the sides of glassware.

2. The solution is allowed to stand without being disturbed. The temperature is allowed to gradually drop, leading to growth of large crystals. The flask should not be placed on a surface (it will shock-cool the solution), but either placed in an insulated jar, or clamped.

Typical problems: Crystals do not form at all (too much solvent), precipitate forms instead of crystals (temperature has dropped too quickly, or an oil forms).

3. The solution is allowed to stand until crystallization is complete.

Typical problems: Crystallization can be a slow process, and impatience can lead to low recovery.

4. The solution is placed in an ice-water bath to lower the temperature even further, and allow more crystals to form. At this point, most crystals should already have formed.

5. The crystals are filtered and air-dried.

How can we tell if a recrystallization has been a success? Simple visual inspection is a good start: The crystals should have shiny surfaces and catch the light. They should appear uniform, and you should have crystals of similar structure and size. A melting point analysis should also show a narrower and elevated melting point range compared to the crude material.

Like any purification technique, recrystallization has some limitations. First of all the compound you crystallize should be a solid at standard conditions. Greases, waxes and oils cannot be crystallized at standard conditions. Secondly, the crude material should be mostly pure. There is not any minimum purity 
standard for any crude material, because the success of any recrystallization depends on the identities of the other constituents and their respective solubilities, but in general the crude material should contain about $80 \%$ of the desired compound.

The crude material is transferred to a suitable crystallization vessel. The crude material is dissolved in a solvent, and gently heated.

The solution is allowed to gently and slowly cool down. Notice the crystals growing in the solution. The solution is cooled to room temperature, leading to the formation of large crystals.

\subsection{DISTILLATION}

Distillation is a purification technique for a liquid or a mixture of liquids. We utilize the difference in boiling points of liquids as a basis of separation. The core of a distillation process, is selective evaporation and condensation of particular components. Our overall goal is to evaporate and condense only one component from a mixture, but to attain this goal, we must allow many, many cycles of evaporation and condensation to take place. This process gradually enriches the vapor phase in favor of the most volatile component. After a sufficient number of evaporation and condensation cycles have taken place, the final condensate contains a liquid that is enriched in the more volatile component.

Distillation is easier to understand if we envision a spesific mixture of two liquids, say diethyl ether and ethanol. The boiling points of the two liquids are $36{ }^{\circ} \mathrm{C}$ and $78{ }^{\circ} \mathrm{C}$, respectively. When we boil this mixture, we observe the following: the entire mixture (both compounds) boils, but the vapor phase is enriched in the more volatile component (diethyl ether). As this vapor mixture rises, cools, and condenses, the resulting liquid is enriched in diethyl ether too. If we attach a column to the flask so that the vapor enters this column, the condensing liquid will be heated by rising vapors, and it will boil again producing a vapor that is even more enriched in diethyl ether. The higher the column, the more times this cycle of evaporation-condensation can be repeated, and the higher up we sample the vapor, the more enriched the vapor phase will be in the more volatile component (diethyl ether). Ideally, with a long enough column, one could obtain a vapor that is nearly pure diethyl ether, and leave behind a liquid that is nearly pure ethanol, the less volatile component.

Let us discuss a typical distillation apparatus (shown above) We start with a flask containing the solution (often called a still pot), which is heated. You can see from our discussion above, that a key feature of a distillation apparatus must be a column, where many cycles of condensation and evaporation can take place. The column can be just a short tube (an "unpacked" column), which is the basis of a simple distillation. This is typically less efficient. The column can be packed with an inert material, which is the basis of a fractional distillation, and typically constitutes a more efficient distillation. The inert material, such as

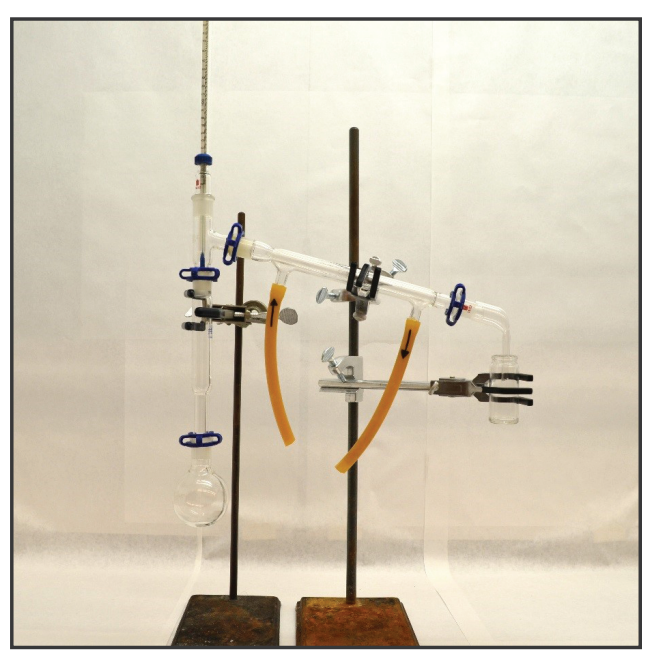

Figure 2.2. A simple distillation apparatus. The round bottom flask (or still pot) is attached to an unpacked condenser. The condenser is attached to a three way adapter that contains the thermometer, and a water jacketed condenser with cold water running through it.

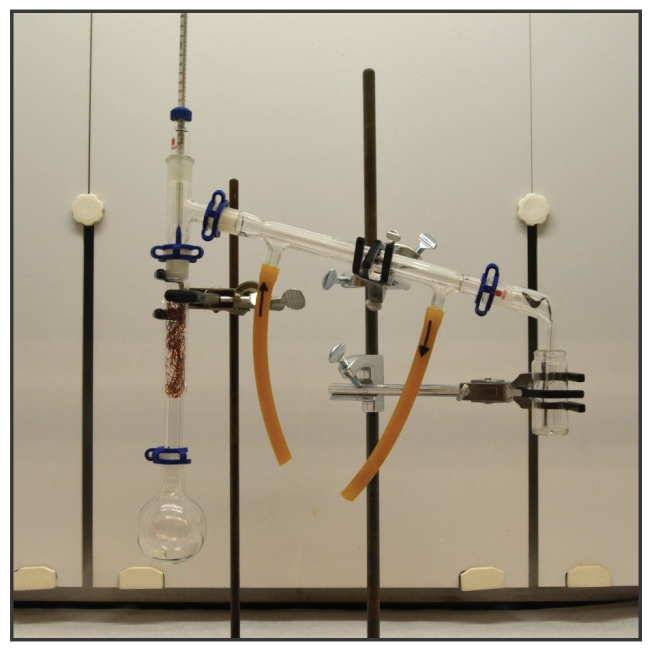

Figure 2.3. A fractional distillation apparatus. The round bottom flask (or still pot) is attached to a condenser packed with copper sponge. The sponge gives a large surface area for the vapor to condense on, leading to a more efficient distillation. 
copper sponge or glass beads, provides a large surface area, thus allowing many more evaporation-condensation cycles.

The column is attached to an adapter. In the top of this connector is the thermometer, which is used to read the temperature of the vapor, just as it condenses. The temperature reading is important, because, at normal conditions, the temperature of the vapor passing through is the same as the boiling point of the substance being collected. Vapor that passes the thermometer condenses in the condenser, a double-walled tube that is cooled by water flowing through the outer layer, and drips into the receiver.

Before moving on, let us look at benefits and drawbacks to a simple distillation versus a fractional distillation. We have already said that the simple distillation is less efficient at separating liquids, because there is a smaller surface area inside the column, but it is usually much faster. For mixtures that contain only one volatile component, a simple distillation can be more than sufficient. The fractional distillation is more efficient, and is suited for mixtures of volatile liquids. The closer the difference in the boiling points, the more demanding the distillation. The drawback is that fractional distillations typically take longer, because we want to achieve pseudo-equilibrium between vapor and liquid throughout this system. Slow boiling and ample time is important to achieve this goal.

Sounds easy, right? What can go wrong? Poor separation is the most likely problem you will face. This means that the fractions obtained are impure, and often contain traces of the other liquids found in the original mixture. We will assume that we are performing a fractional distillation, and that we have several liquids that must be separated. Let us look at some of the technical aspects that are important for acceptable separation, and some key features for the assembly of the distillation apparatus.

\section{Troubleshooting Distillation}

1. The distillation result is poor: the fractions obtained are not of acceptable purity.

Typical problems: Distillation too fast. The components require time to separate. We need many evaporation-condensation cycles for good separation, and pseudo-equilibrium between vapor and liquid throughout the system. If we supply too much energy to the system (i.e., too much heat), we allow even the less volatile components enough energy to keep evaporating. The vapor phase is therefore not enriched with the more volatile component.

A certain amount of time is also required. Allowing the mixture to gently reflux for a while (30 minutes) before gradually increasing the energy supplied to the system through heating is typically a good strategy.

2. You collect distillate, but the temperature reading does not correspond to the boiling point of the component. Typically, the temperature reading is much lower.

Typical problems: The thermometers found in a standard organic chemistry teaching laboratory is not of the highest quality, and they are often mistreated through many terms. They should therefore be calibrated by reading boiling distilled water. Furthermore, the location of the thermometer bulb is essential. If the bulb is too high, the vapor condenses before the thermometer can read the temperature. Typically, the bulb should rest right above the lowest part of the adapter (see the set-up above).

3. Even though the liquid in the still pot is boiling, no distillate is being collected.

Typical problems: Insulation is a key feature to consider whenever distillation does not occur. Have a look at the entire apparatus and see where the vapor has reached, by looking for drops of condensation. Insulating the top of the still pot, as well as the column and top of the three-way adapter can be beneficial. Remember that the vapor must heat the glassware to the boiling point of the condensing liquid before it can evaporate again and rise through the apparatus. Because the distillation is performed in a fume-hood, the constant air-flow inside the hood also cools the apparatus. 
4. Nothing distills but the amount of liquid in the still pot mysteriously goes down.

Typical problems: There is a leak in the system, or several leaks. The vapor is escaping though openings between joints. Make sure that all joints are properly sealed.

\subsection{LIQUID-LIQUID EXTRACTION}

Liquid-liquid extraction involves the exchange of certain compounds between two solvents that are immiscible or only partially miscible. Liquid-liquid extraction is also very commonly used for washing an organic phase, for example to remove inorganic compounds, or to protonate or deprotonate bases or acids, respectively, so they become soluble in the aqueous phase. A very typical extraction flow diagram is shown below, where a reaction mixture is quenched with water, extracted (several times), washed with brine, dried, filtered and finally evaporated to yield a crude product or a pure product.

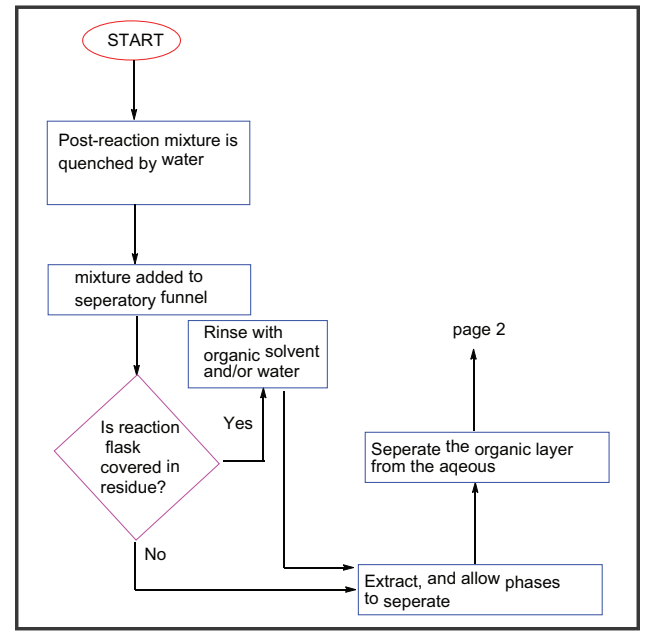

Figure 2.4. A typical flow-chart for an extraction.

\section{FIGURE 2.4. A TYPICAL FLOW-CHART FOR AN EXTRACTION}

Liquid-liquid extraction (we will refer to it simply as extraction from now on) is typically conducted with one aqueous phase (either pure water, or an aqueous solution) and one organic phase. It is important to note that the desired compound (usually an organic molecule) can in theory be in either phase. It depends on both the nature of the compound, but also the nature of the aqueous phase. We will first examine some typical scenarios involving extraction, then move on to discuss technical details to keep in mind.

1. Extraction of neutral compounds. If the desired organic compound is neutral (i.e. is neither acidic nor basic), the extraction sequence usually involves simply extracting with an organic solvent several times.

2. Extraction of acidic compounds. If the desired compound is acidic, we can selectively deprotonate that compound by using an aqueous base. This will pull the deprotonated compound into the aqueous phase. Because very few organic compounds are soluble in water, we can discard the organic phase which now contains any byproducts and/or unreacted starting materials. Acidification of the aqueous phase can precipitate the desired product.

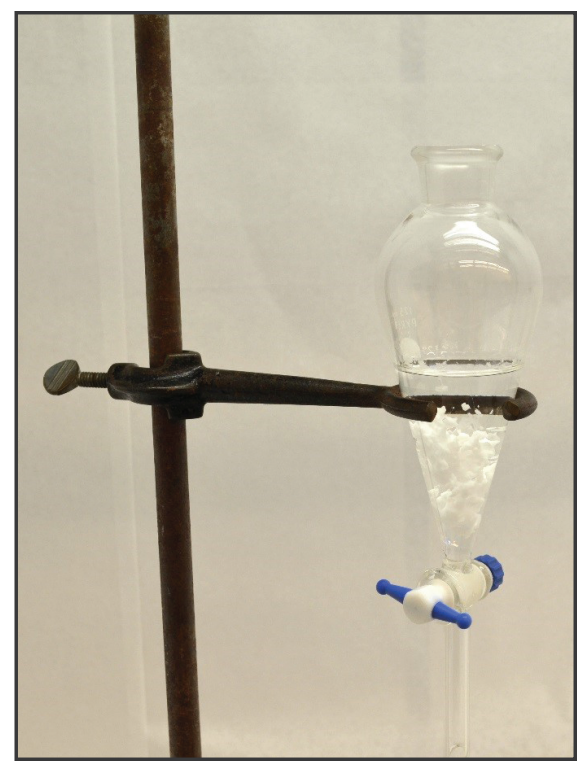

Figure 2.5. Adding $\mathrm{HCl}$ to the basic, aqueous phase leads to precipitation of benzoic acid, here seen as a white precipitate.

3. Extraction of basic compounds. If the product is basic, we can perform a sequence very similar to the acidic compound above. We can protonate the basic compound by using an aqueous acid, pulling the protonated compound into the aqueous phase and discarding the organic phase. Neutralizing the acidic phase will deprotonate the basic compound, which may precipitate, or may require a second extraction with an organic solvent.

The typical apparatus used in an extraction, is the separatory funnel. These come in many different sizes, but the typical size is $100 \mathrm{~mL}$, which you will encounter in several labs at PSU. The funnel is equipped with a top and a nozzle, and the nozzle can be closed or opened with a stopcock. The funnel is narrow at the nozzle, which is a structural feature that allows us to accurately separate the two phases. 
How to perform an extraction. For simplicity's sake, we will assume that the product is a neutral compound.

1. Make sure the stopcock is closed. Add a small amount of water or the aqueous solution you are using. Sometimes the stopcock is improperly sealed, and you will find that it leaks. You would rather know that now, rather than after the organic material has been added.

2. Add the reaction mixture, as well as water if necessary, and the required organic solvent. Make sure that the solutions added do not touch the inside of the top of the funnel, as solids can crystallize here and prevent the stopper to seal properly, leading to leaks.

3. The funnel is closed with a stopper, turned on its head, and shaken very carefully. The stopper is always supported by your hand. The pressure is released after each round of shake by opening the stopcock (point it upwards into the hood, but never towards yourself or someone else), and then closed again. Now the two layers are shaken vigorously. It is imperative that the two phases are mixed, because no compounds will exchange without a good mixing.

4. The funnel is rested in a ring stand, and the stopper removed. The two phases are allowed to separate. The phases are removed by draining them out, one at a time.

5. The aqueous phase can be extracted again, up to three times. The combined organic fractions are typically dried with a drying agent, ${ }^{9}$ then filtered, and the solvent evaporated.

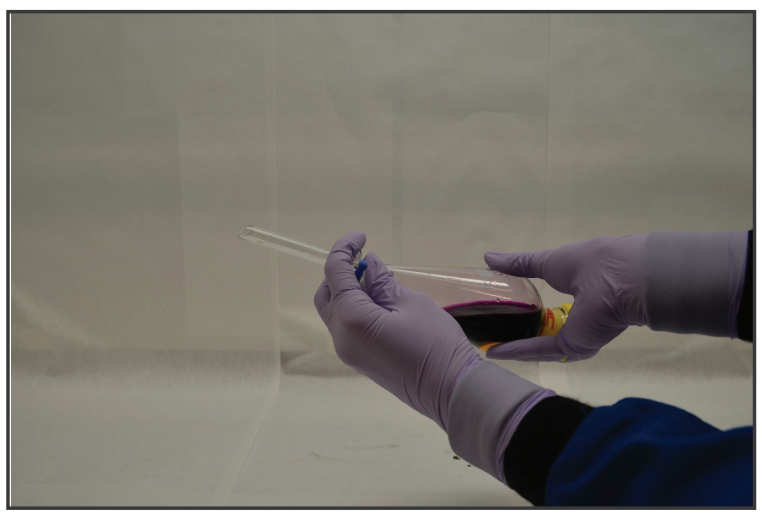

(A) The separatory funnel containing the two phases are shaken vigorously together.

The stopper of the funnel is supported by one hand, and the stop-cock supported by the other hand.

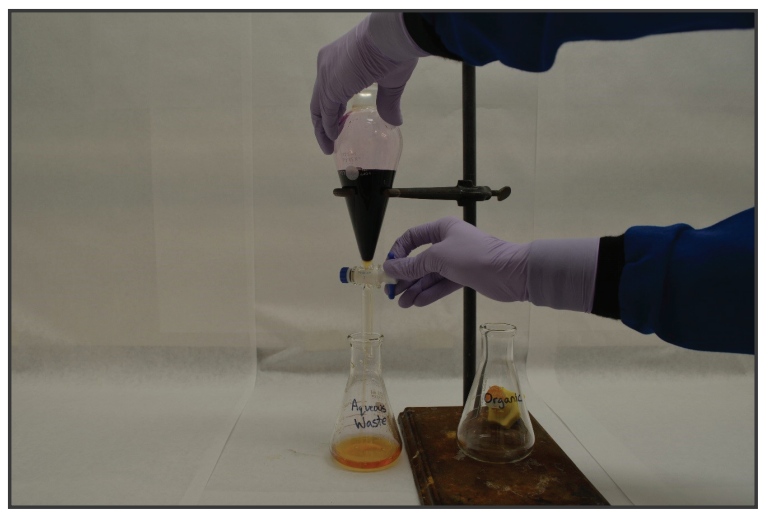

(C) The entire bottom phase is drained, and the stop-cock is carefully closed when all the phase has been removed, leaving the top-phase in the separatory funnel.

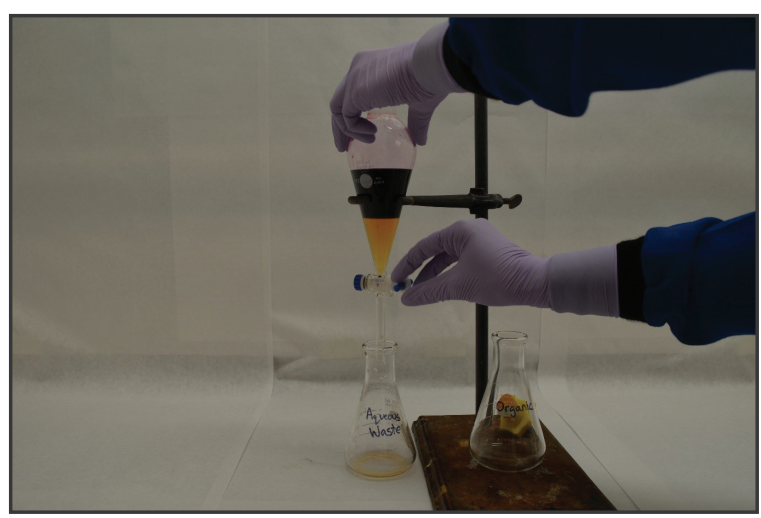

(B) The two phases are separated. The aqueous (or lower phase) is drained into a clean container.

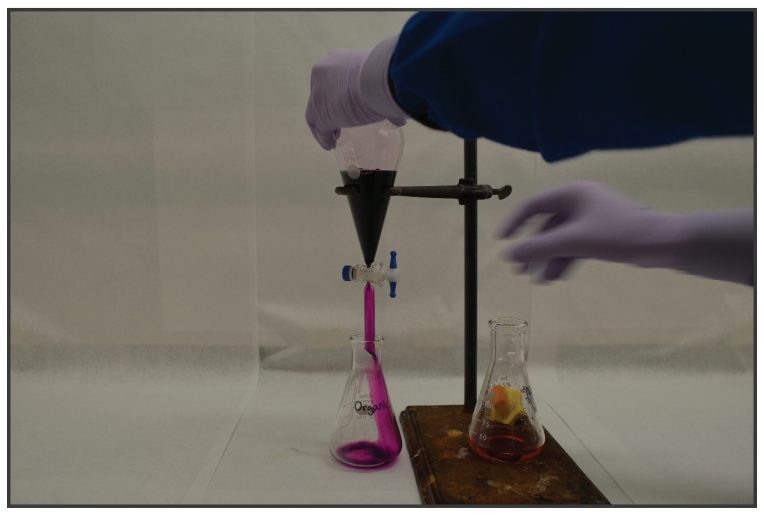

(D) The top phase is then drained into a clean container.

If necessary, either phase can be extracted again, to finish the extraction routine.

Figure 2.6. Key steps in a liquid-liquid extraction 
One common problem with extractions is one that does not arise from improper technique, but from the annoying behavior of certain compounds: they form emulsions. These usually appear between the two layers, and are chemically composed of the two solvents, and other compounds found in the original solution. The emulsion can resolve itself over a few minutes, or be very persistent. In the latter case, it can sometimes be a good strategy to drain the emulsion out in the phase that does not contain the product, and then re-extract it several times.

Let us say that we are extracting an aqueous solution with the organic solvent diethyl ether. Let us also say that the desired compound is organic, and in this example will be dissolved in the organic phase. A very persistent emulsion is formed. If we drain the aqueous phase and as little of the organic phase as we can (while still draining the emulsion out), we can obtain some organic phase without emulsion that is set aside. We then add the phase back to the separatory funnel and re-extract the aqueous phase that still contains some organic phase, as well as emulsion again. This usually disperse the emulsion.

\subsection{TLC -ANALYSIS}

Thin-layer chromatography (or TLC) is a quick, cheap, and reliable way to evaluate the composition of a sample, and the identity of a given compound. The TLC plate works by chromatographic principles: a mobile phase (solvent or solvent mixtures) will climb up the plate material (stationary phase). Compounds that are less polar will wander farther on the plate, because they experience les1s attraction to the stationary material. Compounds that are more polar will wander lower on the plate, because they experience more attraction to the stationary material. This process is separating the different compounds present in the crude sample

Most compounds that we handle in the o-chem labs at PSU should give a single spot on the TLC plate. Ideally, each compound in a mixture will produce a distinct spot so a sample with two compounds will give two different spots, and so on.

An important property of any compound, is its $\mathrm{R}_{\mathrm{f}}$-value (retention factor). In simple terms, this value is an indication of how far up a TLC-plate a compound has wandered. A high $\mathrm{R}_{\mathrm{f}}$-value indicates that the compound has travelled far up the plate and is less polar, while a lower $\mathrm{R}_{\mathrm{f}}$-value indicates that the compound has not travelled far, and is more polar. This value is easily calculated by measuring the distance the spot has wandered, and dividing this by the distance the solvent has traveled. The $\mathrm{R}_{\mathrm{f}}$-value is dependent on both the compound and the solvent used for development. If we analyze two compounds and they give the same $\mathrm{R}_{\mathrm{f}}$-value with the same solvent system, the two compounds are most likely identical.
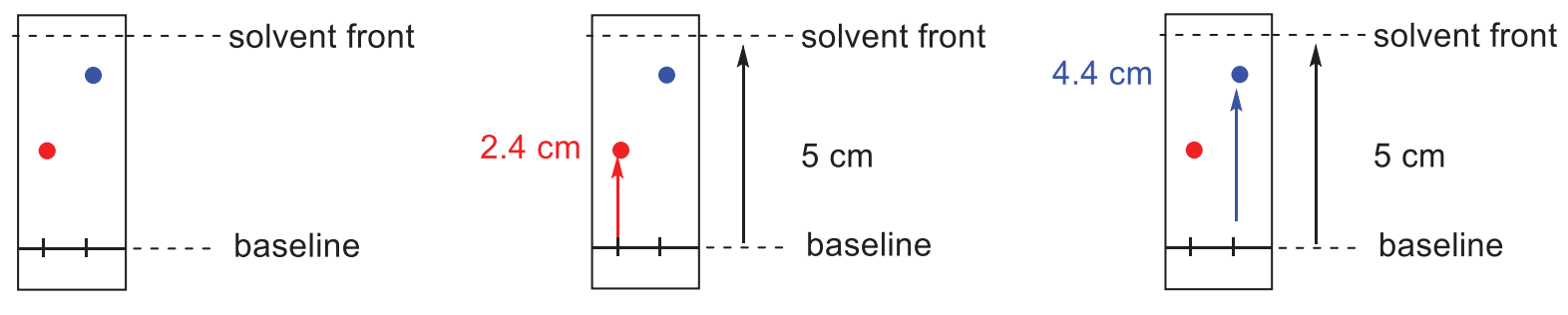

$$
\mathrm{R}_{f}=2.4 / 5.0=0.48
$$

$$
\mathrm{R}_{f}=4.4 / 5.0=0.88
$$

Figure 2.7. Calculation of the $\mathrm{R}_{\mathrm{f}}$-values of two spots.

The apparatus for doing a TLC-experiment is very simple. We need a developing chamber where the mobile phase is kept. The chamber should be sealed, and although there are special TLC-chambers one can use, a beaker covered by a watch glass to create a seal often suffices. 


\section{How to prepare a TLC plate:}

1. A horizontal line is drawn at the bottom of the plate $(5-8 \mathrm{~mm}$ from the bottom edge $)$ and another horizontal line is drawn at the top of the plate ( $8 \mathrm{~mm}$ from the top edge). A gentle soft and a very soft pencil must be used because too much pressure or harder pencils will crack the very thin coating on the plate, leading to unacceptable TLC results after development (ink pens should never be used because the ink will wander up the plate).

2. Vertical ticks are drawn on the bottom line, depending on how many samples are analyzed. These are usually labeled (A, B, C, for example)

3. Solutions are prepared of all the samples that must be analyzed. These must neither be too concentrated, nor too dilute and a concentration of about $5 \%$ is a good starting point. The samples are applied to the ticks with capillary tubes without damaging the coating on the plate.

4. The TLC plate is placed into the developing chamber, and care is taken to make sure that the bottom edge of the plate is perpendicular to the solvent. If not, parts of the plate will overdevelop, and we will get poor resolution and unclear results.

5. The chamber is sealed with the watch glass and the solvent front is allowed to climb up to the topmost line. We then remove the plate and allow it to dry before we observe the plate under UV-light.

We will discuss two common ways to use a TLC- analysis. The first is to verify the identify of a compound. A quick TLC analysis can be used to identify whether or not an unknown compound is the same as another known compound. We typically make three applications: one of the unknown sample, one of a stock (known) compound, and a third with one spot of both (called a co-spot). If we find that the two spots have the same $\mathrm{R}_{\mathrm{f}}$-values, and the third spot only shows one spot, the two compounds are identical.

The second common way to use a TLC- plate, is to monitor a reaction. We can use TLC-analyses to see whether or not a reaction has gone to completion. We typically see the disappearance of the starting material(s) and the emergence of a new spot that corresponds to the product. We usually have to make controls as described above, with spots of the starting material(s) to learn their $\mathrm{R}_{\mathrm{f}}$-values.

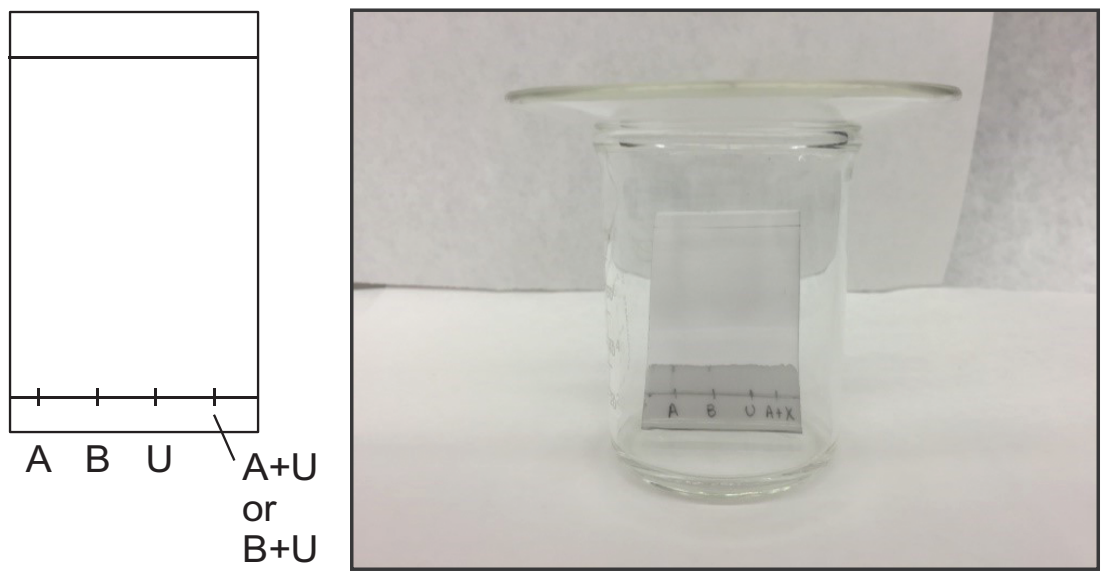

Figure 2.8. A labeled TLC plate. 


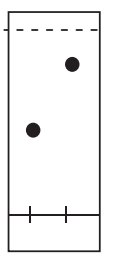

(A) The plate is good.

The two spots are small and clearly defined.

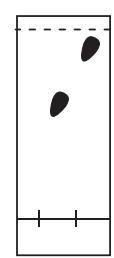

(B) The plate is not good.

The spots have "drifted" which most likely was due to the plate not being perpendicular to the solvent when it was inserted into the chamber

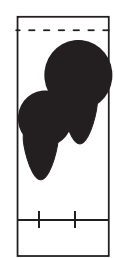

(C) The plate is not good.

The two spots are large, because the sample was too concentrated.

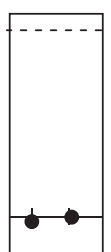

(D) The plate is not good

The spots have not wandered because the solvent system used, was not polar enough.

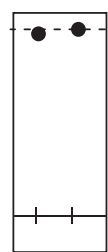

(E) The plate is not good

The spots have wandered to the solvent front, because the solvent was too polar

Figure 2.9. Various TLC scenarios.

Like any of the techniques we have discussed in this chapter, TLC-analyses require a fair amount of care to get right. The most likely problems can be traced to the application and preparation of the TLC-plate. Let us have a closer look at some TLC plates that are of inadequate quality and their causes.

\subsection{SUBLIMATION}

Sublimation is a purification technique for solids and in the context of this book, for organic compounds with lower melting points. Sublimation describes the process of a solid becoming a gas, without passing through the liquid state. The gas phase is then typically crystallized on a cold surface. However, many sublimations require reduced pressure, and we will primarily focus on that variation of sublimations. The reason for many sublimations happening under reduced pressure, is because the sublimation point is decreased with decreasing pressure. To allow organic compounds to sublimate, and not simply melt, a reduced pressure is often necessary.

A crude, but efficient sublimation apparatus can be made from of a filter flask, where the side neck is connected to an aspirator or vacuum trap, and the neck is equipped with a cold finger (a tube with ice water, or other cooling material). The impure solids are placed in the bottom of the filter flask, and the cold finger is inserted. While an aspirator or pump is reducing the pressure inside the filter flask, the material is carefully heated on a hot plate (take care to avoid melting or boiling the material). The organic compound will start to sublime forming a gas. Once this gas reaches the cold finger, it will immediately crystallize on the cold finger, where it can be collected.

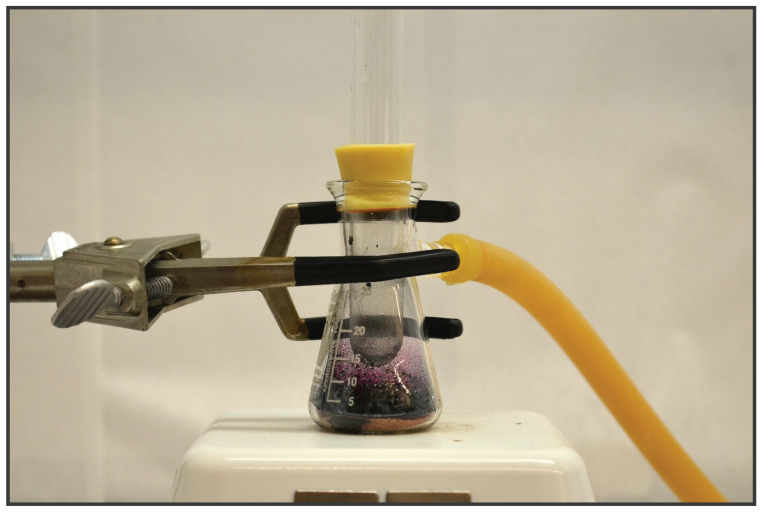

(A) A filter flask is clamped. The side arm is attached to a tube connected to a water aspirator, leading to reduced pressure inside the flask.

The cold finger is fitted through the neck. There is ice-cold water in the cold finger.

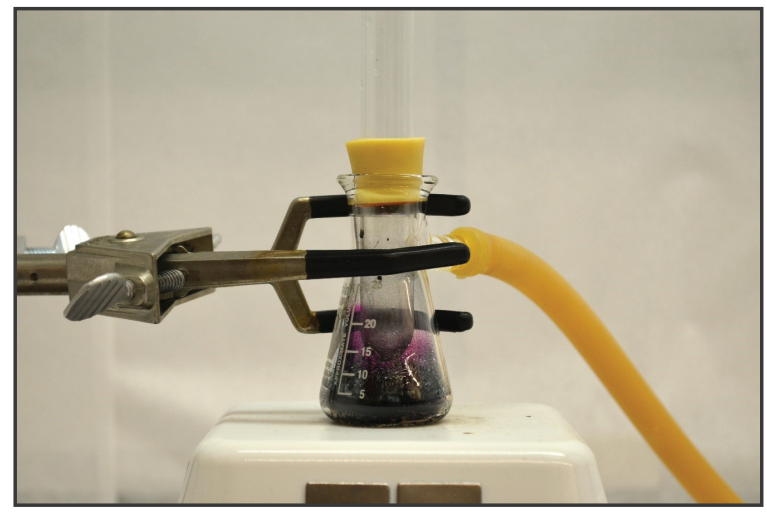

(B) The filter flask is heated. The crude material starts to sublimate. Notice the rising purple vapor. 


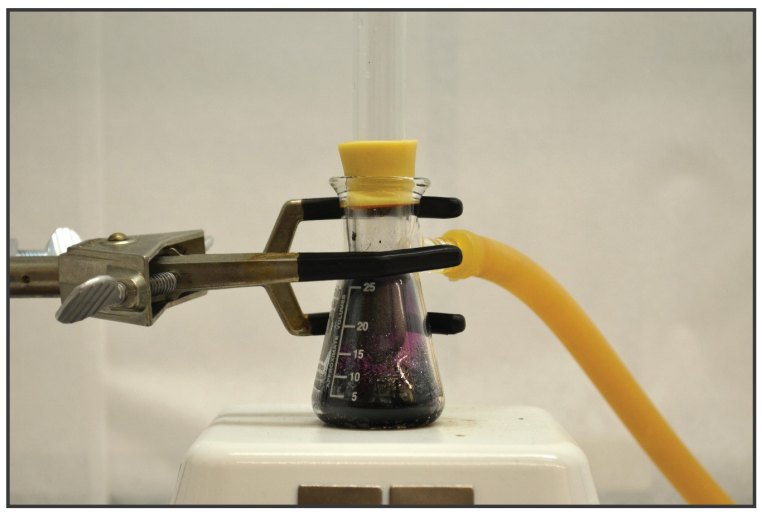

(C) As the temperature rises, more crude material sublimates. Inside the filter flask, the vapor reaches the cold finger.

The vapor then crystallizes immediately.

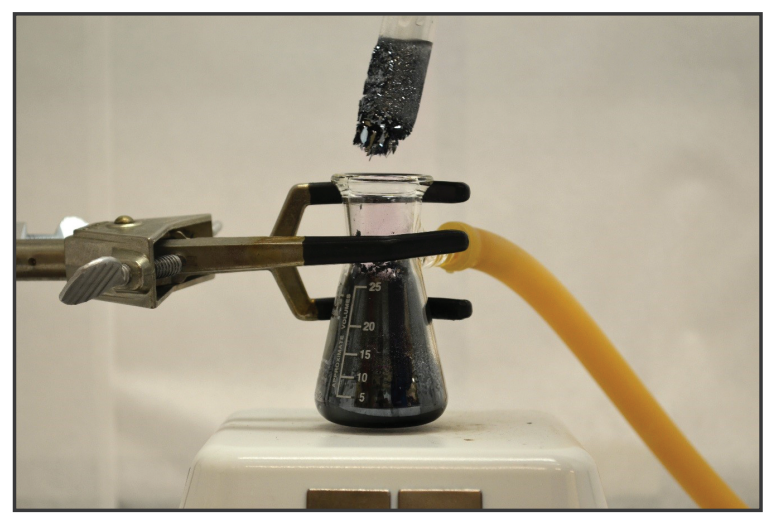

(D) Once the sublimation is complete, the cold finger is carefully removed.

Notice the crystals that have grown on the cold finger.

This can be scraped off, and quantified.

Figure 2.10. Key steps for sublimation

\section{Troubleshooting sublimation}

1. The crude material does not sublime.

The most common reasons are insufficient heat, and/or the pressure is not low enough.

2. The sample sublimes, but the material crystallizes on the sides of the filter flask, and not on the cold finger.

The bottom of the flask might be hot enough, but the sides are not. The material is allowed to sublimate, but cannot reach the cold trap and is able to crystallize earlier. Insulation of the lower part of the filter flask can be appropriate.

3. I don't know where the sample went!

The crude material was added, but during the process of heating the flask, the material is gone, and there are no crystals. There can be several reasons, but the most likely contender is that the cold finger did not efficiently work as a trap. Lowering the position of the cold finger is a good solution.

4. The crude material is boiling!

The system can be too hot, and less energy should be supplied to heat the system. Potentially the pressure should be reduced.

5. The product crystallizes on the cold finger, but looks pasty and wet and not crystalline.

The ice cold water in the cold finger has been in the finger for too long. The cold surface has started condensing water from the atmosphere, and once the product starts to reach the cold surface it is met by water. In general, the ice cold water should be added to the cold finger right before the sublimation starts to eliminate this problem. 
GETTING YOUR HANDS DIRTY:

CHEMICAL HANDLING, WASHING, WASTE AND SAFETY

\section{INTRODUCTION}

In chapter 1 we discussed some of the possible dangers associated with exposure to the chemicals used in organic chemistry labs. In this chapter, we will examine techniques to reduce such exposures. We will also discuss waste and waste management, safety in the lab, and lastly the mental health of the student during the lab experience.

\subsection{GLOVES: THE GOOD, THE BAD AND THE UGLY}

When you start to think about ways to handle chemicals to ensure your safety, you likely think about wearing gloves to minimize contact between your skin and the chemical. Let us get one thing straight immediately: the gloves ${ }^{10}$ may offer only limited protection. This is an important fact because a common misconception is that the gloves you find in lab effectively protect the skin from solvents and other substances handled. This is simply not true.

To give you one example, the nitrile gloves that are typically made available are easily penetrated, and eventually broken down, by two common (and hazardous) solvents, acetone and dichloromethane.

There are several excellent online resources to find information about the permeability of various solvents and reactants. ${ }^{11}$

Another reason we wish to alert you to the limitations of lab gloves, is that wearing them can provide a false sense of security, and this can be very dangerous. Imagine you are wearing gloves, First, you now think that they are protecting you. You are perfectly safe. As a result, you might be less careful, or you may pay less attention to the risks involved in tyour experiment. Not only that, once you put on gloves, you probably pay little attention to how you handle the gloves themselves, what we call "glove hygiene".

Common examples of poor glove hygiene (things you must always avoid) include: touching (and contaminating) your face, skin, hair, or clothes with your gloves, touching (and contaminating) objects like phones, pens, and notebooks, that you will use outside the lab, failing to check gloves periodically for leaks and cracks, and failing to remove gloves each time you leave the lab

In general, gloves should only be worn for short periods of time, and exchanged routinely, especially whenever a chemical has been in contact with them. The contaminated exterior of the gloves should never come in contact with your skin. Whenever you are done with your gloves, you should remove them in a safe manner and wash your hands (never use bare hands to remove gloves).

10 The most commonly employed gloves in many teaching labs, are nitrile gloves. There are many different gloves, in general the more cumbersome they are to wear, the better protection they offer. Unfortunately, there are no gloves on the market that are both practical and offer protection against most organic chemicals encountered in undergraduate organic chemistry labs. pdf

Ansell has a very good overview. https://www.ansellpro.com/download/Ansell_7thEditionChemicalResistanceGuide. 


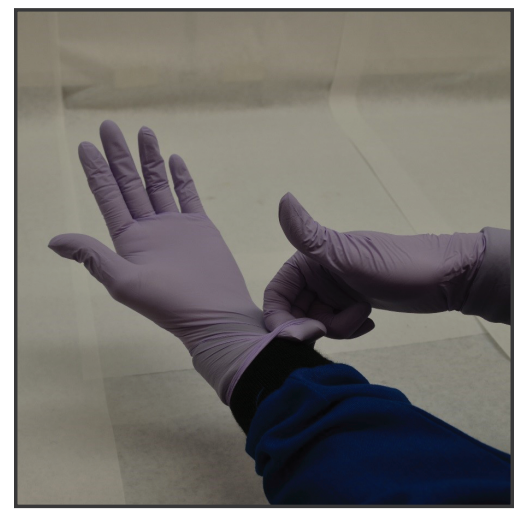

(A) Start by hooking a finger into the outside of the glove.

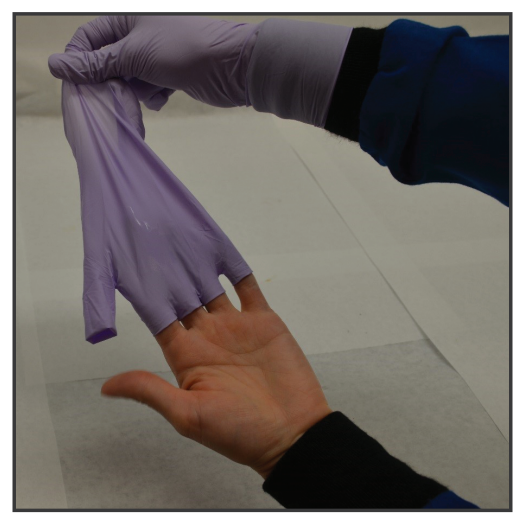

(B) Gently pulling upwards, remove the first glove.

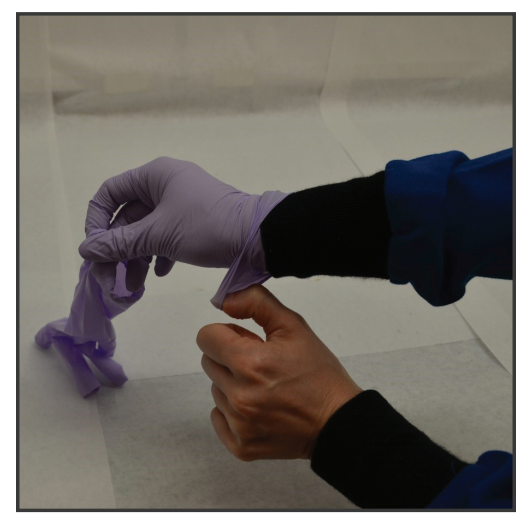

(C) Using your free hand, hook your thumb under the second glove.

Pulling upwards, remove the glove completely.

Figure 3.1. A safe way to remove laboratory gloves.

\subsection{GETTING THE CHEMICALS TO YOUR REACTION FLASK: POURING, SYRINGES AND WEIGHING}

Laboratory chemicals are typically stored, and dispensed, in a variety of containers and locations. Therefore, early hurdles in every experiment are 1) figuring out how to obtain the desired amount of the chemical, 2) figuring out how to transfer this material safely from the location where it is obtained to the location where it will be used, and 3) figuring out how to put the materials in the apparatus.

Solids: Solids are usually stored in wide-mouthed bottles and weighed before use. The solid is weighed using a weigh boat, or a piece of weighing paper, and it is usually possible to transfer the solid from the bottle to the boat (or paper) using a clean spatula or lab scoop. A good laboratory practice is to remove the weigh boat (or paper) from the balance each time you add solid to it, and then return the boat (or paper) to the scale. This practice reduces the likelihood that any chemicals will get spilled on the balance.

Liquids: Liquids are typically more difficult to handle, and the appropriate method will depend on how much liquid is needed and the specific properties of that compound. In most cases, it will be safer and more convenient to obtain a specific volume instead of weighing the liquid. For reagent quantities, a pump system is often employed to deliver calibrated volumes, or a graduated cylinder can be used. Solvents, whether needed for reactions or extractions, tend to be used in larger quantities and a graduated cylinder will nearly always be more conventient.

\subsection{WASHING YOUR GLASSWARE}

Cooking a dinner creates dirty dishes and doing an organic chemistry lab experiment creates dirty glassware. Cleaning your glassware properly is an important aspect of the lab experience. This should be a priority, as impurities or trace amounts of chemicals from previous labs can ruin or complicate your experiment. Starting with visibly dirty or contaminated glassware is also considered poor lab practive, as the outcome of the reaction cannot be trusted: was the outcome due to the reagents you combined, or was it in some way influenced by the contaminants?

We will cover two good cleaning protocols, one for glassware that has been used for organic samples, and one for inorganic solutions.

A good cleaning protocol for glassware that has contained organic solutions or samples: the glassware is rinsed with acetone in the hood, and the contaminated acetone solution is then poured into the organic waste. The glassware is then allowed to air dry. If the glassware is still dirty, try this: wash with soap and 
water, rinse with distilled water, then rinse with acetone again, and air-dry.

This might seem like a very involved process, but in most cases the glassware is perfectly clean after the initial acetone rinses and requires no further cleanings. Also, by performing an acetone rinse first, it is usually safe to remove still dirty glassware from the hood for further cleaning because the acetone rinse will most likely have washed away potentially harmful organic materials. Then, when you wash with soap and water, you do not need to worry about working with potentially harmful organic materials outside of the hood, or pouring them into the drain.

Glassware that has contained only non-hazardous inorganic solutions (such as dilute solutions of $\mathrm{NaHCO}_{3}, \mathrm{HCl}, \mathrm{NaOH}, \mathrm{NaCl}$, and so on) can be rinsed with water in the sink, and then soap, if necessary, washed with soap and water. The glassware is then lastly rinsed with distilled water and allowed to air dry.

\subsection{WASTE}

Waste is a philosophical concept, because we determine, somewhat arbitrarily, when a solution or a substance has become waste. Once you no longer have a use for a chemical, it becomes a 'waste' that must be properly disposed of.

You might argue that this is a flawed concept (pouring $20 \mathrm{~mL}$ of $\mathrm{NaCl}$-solution into a beaker, for example, does not change it from said solution to waste), but we employ this waste policy to ensure that we never contaminate any of the stock solutions, or samples.

In the PSU organic chemistry labs, we have three distinct types of waste containers:

Organic waste: Anything organic goes in this waste container, including organic solvents, solids that are left over from reactions or analyses, products obtained, organic extraction solvents and so on.

Aqueous waste: All aqueous solution used or obtained are poured in this waste container. You might be tempted to pour it straight in the drain, but the solutions could have been in contact with organic material. Let us for example say that you wash an organic extract with a saturated $\mathrm{NaCl}$ solution (brine), and you drain it out. This has been in contact with organic solvent and material, and even if we consider a relatively low solubility, the aqueous solution will still contain some organic molecules.

Solid waste: Solids, such as drying agents, are disposed of in these jars, but so are TLC plates, and any paper tissues that have been in contact with your fume hood.

Obviously we provide these waste containers as part of our safe laboratory hygiene practices, and therefore we wish to train our students in the proper disposal of chemicals, but you might ask, "What are the implications of not following these procedures?"

Let's look at the alternative.

As we have covered earlier, most organic chemicals have a number of safety hazards associated with them, either immediate, long-term, or both. Failing to dispose of these chemicals properly might create health hazards for the workers who unknowingly come into contact with these chemicals. Also, many chemicals that might seem relatively safe in a lab environment, are bio-accumulated by other organisms. These chemicals can produce disastrous effects for when they enter the environment beyond the lab. Aquatic organisms are especially vulnerable, and many organic chemicals have harmful toxicity with long lasting effects for these organisms.

The handling and disposal of chemicals is therefore not only an important aspect of the day-to-day work in the lab, but also for the environment, ecology, and the workers and other organisms that surround us. 


\subsection{WHAT CAN GO WRONG? SOME SAFETY SCENARIOS TO CONSIDER.}

Even with full awareness of safety, and the utmost of care, accidents can happen. It is important to consider some safety issues that can arise, and discuss how best to approach them. One of the most important things to note, is that your health is the most important consideration for everyone in the lab: instructors (TAs and professor in charge), support staff and lab mates. If you have an accident, you must alert your TA immediately. Do not try to remedy the problem on your own, and do not try to cover it up. Every second may make the difference between a good and a bad outcome when an accident has happened, and it is imperative that you get the help and assistance you need, immediately. Your safety is always more important than your pride.

\subsection{STRESS AND ANXIETY}

It is not unusual to feel worried and anxious about your performance in the organic chemistry labs. Your concerns might range from anticipated difficulties in the lab (handling of chemicals, dangerous substances) or just your desire to do a good job.

Here are a few strategies that can help mitigate any feelings of anxiety and stress:

1. Plan ahead. It sounds simple, but as we have seen from the first chapter, planning is the most important step before undertaking an experiment. Remember to visualize the experiment prior to the lab so you are mentally prepared. This will prepare ou for the things you need to do and the decisions you will need to make.

2. Calm yourself down. If you feel overwhelmed or anxious, a very effective trick is to make sure your apparatus and chemicals are taken care of, and then simply taking a step back and taking five slow, deep breaths. This calming exercise can mitigate any imminent feelings of discomfort, and help you reassert yourself before moving on.

3. Communicate with your TA. If you find yourself feeling overwhelmed or deeply uncomfortable while in the lab, communicate this to your TA. Your TA will help secure your apparatus and material, and potentially have a quick chat about your current state of mind. If necessary, the TA, student and professor will work together to find a plan to assist the student.

4. Take a break. If the experience simply gets overwhelming, tell your TA, and take a short break. Leave your lab coat and goggles behind, and go out in the hall, or take a bathroom break.

Remember, lab should be a positive, engaging and interactive learning experience. Although it is natural (and sometimes even helpful) to compare yourself to peers, experiments by their very nature have a variety of outcomes, and you can learn something from all of them. The primary objective in the lab is to focus on what you are doing. ${ }^{12}$

It is also worth mentioning that we have seen incorrect advice spread in the lab through this interaction. This happens because of discussion, when one individual presents an idea or concept to his or her peers, and the other students accepts it. Although someone is free to give their opinion, it is always worth taking it with a grain of salt, and ask your TA if you are unsure. 


\section{INTRODUCTION}

We have talked about preparation, safety and all the processes that should happen before an experiment. Now we will move on to the actual experiment itself. The aim of this chapter is to contextualize the organic chemistry experiment, and discuss the different phases of the experiment. You will find that most experiments follow the same general outline, which should make it easier to prepare.

\subsection{TYPICAL FLOW OF AN ORGANIC CHEMISTRY EXPERIENCE}

There are typically three phases associated with an organic chemistry experiment:

\section{Phase 1: Set-up and reaction}

The reaction apparatus is set up, and the reactants and solvents are measured and mixed together. The reaction mixture is stirred, and heating the reaction mixture is often necessary.

\section{Phase 2: Work-up and purification}

The reaction mixture is often quenched with water, and then work-up is performed. Work-up can be a combination of the purification techniques discussed in chapter 2 , such as extraction, filtration and/or distillation. The aim of this phase is to separate the main product from the other components, and isolate it in high purity.

\section{Phase 3: Characterization}

The obtained product is analyzed by means of different techniques, such as melting point analyses, and IR spectroscopy. The aim of this phase, is to obtain information about the identity and the purity of the product.

Keeping this organization in mind can be valuable, as it firmly cements the purpose of the experiment. Each phase has distinct purposes, which all funnel into the overall goal of the experiment.

The set-up and reaction phase is all about setting up the conditions of the experiment. We want a certain chemical reaction to take place, and must add reactants in exact proportions, with solvents and potential catalysts. The reaction conditions, i.e. the temperature, the time and other parameters, are usually defined at the outset. This phase is also crucial in determining the success of the experiment. Without weighing out correct quantities of reactants, for example, or heating the mixture to the correct temperature, the desired reaction may not find place, or competing side-reactions might happen instead

The work-up and purification phase usually involves the most skill and technique. After the reaction stage, we have a mixture of potentially unreacted starting materials, products, solvents and byproducts. At this stage, a series of purification techniques are employed to isolate the product, from this mixture. 
The analysis and spectroscopy phase has one aim: to establish the identity and the purity of the compound prepared. Several techniques are involved, usually melting point analyses (if the product is a solid), and IR spectroscopy. In some cases NMR spectroscopy is also used in the teaching lab.

Please notice that while information about the final purity and identity of the compound is obtained in phase 3 , the actual outcome of the experiment is determined by what took place during the reaction and the purification stage, phases 1 and 2. We will explore this matterfurther in the next subchapter.

\subsection{WHY DID MY EXPERIMENT FAIL TO WORK THE WAY I EXPECTED?}

Experiments fail all the time to work in the ways we expect, especially in a teaching lab. Countless students each week in numerous colleges and universities do not get their product. ${ }^{13}$ Why not?

The answer is complex, but we will try to go through some common reasons experiments fail in the teaching labs.

Previously, we said that the outcome of the experiment is set in phase 1 , and that very often the purity of the product is set in phase 2. Let us examine these statements closer. What can be the reason for a failed experiment, an experiment in which no product is obtained?

Is it because the chemical theories underlying the experiment are fundamentally wrong? Or is it because the procedure given to the students contains a fatal error? If either of these possibilities were true, we would expect the experiment to fail for the entire class because these are fundamental flaws in the design of the experiment and should apply to everyone equally. If, however, we see that the experiment works for some students and not others, we must seek an explanation at the level of the individual, i.e., we must assume that the different outcomes reflect different choices that individual students made.

If absolutely no product is obtained, the mistake or error can usually be traced back to stage 1 . Very typical mistakes (that the student might not have been aware of) are calculation errors and/or errors in measuring out reactants. A misplaced decimal point, e.g. $0.1 \mathrm{~g}$ versus $0.01 \mathrm{~g}$ is easy to overlook. Other common errors are improper heating during the reaction stage, and letting the reaction continue for a wrong period of time. Another error that we see quite often, is use of the wrong reagents. Chemical names are strange, and it is easy to overlook subtle differences such as "conc." versus "6M" sulfuric acid, or acetic anhydride versus acetic acid. The reagents in each pair sound very similar, but may react completely differently. Of course, with so many possibilities for error, it can be very difficult to backtrack and find an error after the experiment is complete.

Simple misunderstandings often occur, even with a very carefully written procedure, and a very careful TA. To give you an idea of the level of involvement required in designing lab manuals, all of the PSU organic chemistry labs have been piloted at least three times, by three different people. The manual is constantly updated and refined, and even after several terms, minor changes are still implemented. This means that people read and interpret differently. Something that might seem very clearly worded to you might be very confusing to someone else. In this case your TA will aid you in figuring out what has gone wrong.

Sadly, even when a reaction has been performed properly, it is possible for things to go wrong in stage 2 , the work-up and purification. In fact, this is often a greater source of trouble because purification, by its very nature, produces two things: the desired (pure) material and the undesired contaminants. Unless one pays careful attention, one can easily toss out the purified product and hold on to the contaminants. Also, as you probably noticed in chapter 2, purification procedures can be complicated and there are many ways for them to go astray.

\footnotetext{
13 There are no overall statistics as far as I am aware, and this is simply based on my experience, but usually in a class of 20 students, anywhere from 1-5 of them have experiments fail in any given week, this often happens for unknown reasons. Happily, only once in a while is a student so unlucky to have two failed experiments in a given quarter.
} 
Getting two phases confused during a liquid-liquid extraction, for example, or failing to get crystal formation during recrystallization, are examples of mistakes that can prevent you from obtaining product.

\subsection{THE DEEP UNDERSTANDING}

I believe that the following will not be a surprise to anyone, but it is perhaps the primary reason why students suffer through sub-par lab experiences: lack of understanding. One piece of advice I usually give is to make sure that you understand each step in the procedure.

A chemical procedure is in many ways like engineering: every element serves a purpose, and has a specific function. A very fulfilling and rewarding exercise is to make sure that you have this correlation firmly in your mind before you enter the lab. This will give you a deep understanding, and make the outcome of your experience more positive, both scientifically but also personally.

Do you really understand why we added the base in step 3? Do you really understand why we shake the separatory funnel, or why we add the anhydrous sodium sulfate? Do you really understand why we distilled the product, and why we did not recrystallize it?

A key feature of surviving (and enjoying!) an organic chemistry lab experience is therefore to attain a deeper understanding. That way, you will see how the elements of the procedure come together, and how each aspect has a vital and important purpose. This can be a valuable way to prepare, and to make sure that you are stacking the odds in your favor, to make sure that the experiment goes well.

\subsection{TAKING NOTES AND USING THE NOTEBOOK}

One of your key tools in the lab, is your notebook. This is equally true of real research situations, where a scientist takes careful notes during an experiment. The point of the notebook is to collect data on your procedure, as well as observations you make. In theory, you can fill the entirety of a notebook in only one experiment, if you uncritically write down anything that you can think of. The key to using the notebook is therefore to collect not only data, but relevant data. This data will be imperative for you when you compile your laboratory report later.

The distinction between irrelevant- and relevant data can be challenging, but we will cover some situations here. The most important aspect to remember, is that relevant data provides proof and indication of the success and outcome of an experiment.

1. Numbers and units. Masses and/or volumes of starting materials, catalyst solutions and reaction solvents are always important. They will deviate slightly from the quantities given in the procedure, and you should record what you actually use because you will also use these numbers later. Many other numbers are unimportant. The amount of drying agent (you add enough), the amount of water in your water bath (you add enough), the setting on your hot plate, the size of the Erlenmeyer flask you collected your organic extract in, and the size of the filter paper are not important.

2. Logic. When you are asked to write a procedure as part of your lab report, you must provide a text describing what you did. The procedure in your report must match the information in your notebook; you should never draw on the lab instructions to fill in missing gaps. That said, many essebtuak steps are unnecessary to note. For example, if you filter a solution using a Buchner apparatus, it is not necessary to make note of the fact that you clamped the neck of the filter flask, and wet the filter paper before you turned the water aspirator on. These are standard steps that are automatically included in "the solution was filtered using Buchner filtration" is sufficient.

3. Observations. This is in many ways a very difficult category, because you can make an infinite amount of observations over the span of an experiment, but many of them are secondary or irrelevant. For example, a reaction mixture might change color from clear to yellow, and finally to brown. That may or may not be important, 
depending on the specific experiment. Some color changes indicate the formation of key intermediates and provide useful guides to an expert reader. Other color changes may not be so readily interpreted, but may still be useful because they serve as landmarks of the chemical changes that are occurring. Always pay attention to your experiment, as small subtle changes can be important. 


\section{HOW TO INTERPRET YOUR RESULTS}

\section{5}

\section{INTRODUCTION}

Upon completion of the lab, you will move into a new phase of your lab experience. You now must now carefully evaluate your results with care and interpret them in a logical way. In this chapter, we will focus on the interpretation of the three most important types of results.

\subsection{WHAT DOES IT ALL MEAN?}

We have already said that any experiment has some inherent goal.

Many organic chemistry experiments are performed for the express purpose of obtaining a target compound. The obvious goal of these experiments is the target compound, but how can you satisfy yourself that the goal has been achieved? Of equal importance to the target are data and measurements that prove the identity and purity of the target. Some results that indicate the identity and purity of a compound are IR spectroscopy, melting point analysis, and TLC-analysis. As you will see, these results provide different kinds of information so you will normally collect more than one type of data. Let's go through some typical scenarios, that illustrate the ways that data can be interpreted.

\subsection{IR -SPECTROSCOPY: THE WORKHORSE}

IR-spectroscopy is perhaps the most frequently used technique in the organic chemistry labs at PSU. It is routinely used to identify products and to verify that an experiment has succeeded. One limitation of IR -spectroscopy that is worth remembering is that while the data can be used to distinguish clearly between many functional groups, the data is nearly useless for distinguishing between structural isomers, or characterizing carbon skeletons that contain the same functional groups

An IR -spectrum routinely shows peaks from the range of 3600 to $500 \mathrm{~cm}^{-1}$.

IR -frequencies correspond to the frequencies of molecular vibrations. Molecules vibrate at many frequencies, because each vibration involves a particular group of atoms, and a particular motion such as stretches, bending, wagging etc. The same group of atoms will often vibrate at the same frequency, regardless of the surrounding molecular structure. This fact enables us to assign specific peaks to specific functional groups.

There are several useful sources of IR-spectroscopy correlation tables, and a simplified version is supplied here. Although not very extensive, this table gives you information about the most important functional groups encountered in the organic chemistry labs at PSU.

Something that is worth noting is that many functional groups undergo two types of vibrations and produce peaks in two or more areas of the IR- spectrum. In these cases we should look for, and find, all of the 
Table 1. Simplified IR spectroscopy correlation table

\begin{tabular}{|c|c|c|}
\hline Bond & $\begin{array}{l}\text { Absorption } \\
{\left[\mathrm{cm}^{-1}\right]}\end{array}$ & Comments \\
\hline $\mathrm{O}-\mathrm{H}$ & $3600-3400$ & $\begin{array}{l}\text { The peak is usually broader than most, and its intensity and frequency vary with concentration. If present, also } \\
\text { look for C-O peak. }\end{array}$ \\
\hline $\mathrm{C}-\mathrm{H}\left(\mathrm{sp}^{2}\right)$ & $3080-3010$ & Alkene or benzene. If present, also look for $\mathrm{C}=\mathrm{C}$ peak. \\
\hline $\mathrm{C}-\mathrm{H}\left(\mathrm{sp}^{3}\right)$ & $2960-2820$ & $\begin{array}{l}\left.\text { If the sample is a DCM-solution, you will find a very strong solvent peak here that obscures any C-H ( } \mathrm{sp}^{3}\right) \text { peaks } \\
\text { that might be generated by the target compound. }\end{array}$ \\
\hline C-H (aldehyde) & 2720 and/or 2820 & Very characteristic. If present, also look for $\mathrm{C}=\mathrm{O}$ peak. \\
\hline $\mathrm{C}=\mathrm{O}$ (carbonyl) & $1750-1680$ & $\begin{array}{l}\text { Very characteristic. A large number of functional groups contain a carbonyl group, so, if present, also look for other } \\
\text { peaks that might identify the functional group }\end{array}$ \\
\hline $\mathrm{C}=\mathrm{C}$ & $1680-1600$ & Alkene or benzene. \\
\hline $\begin{array}{l}\mathrm{C}-\mathrm{O} \text { (alcohol, } \\
\text { ester, ether) }\end{array}$ & $1300-1100$ & $\begin{array}{l}\text { Complementary bond in alcohol, esters and ethers (but not only those bonds). If an } \mathrm{O}-\mathrm{H} \text { peak is present, this } \\
\text { might indicate an alcohol or carboxylic acid group, while the presence of a carbonyl peak and absence of } \mathrm{O}-\mathrm{H} \\
\text { peak can indicate an ester group. The absence of both } \mathrm{O}-\mathrm{H} \text { and carbonyl peaks would indicate an ether group. }\end{array}$ \\
\hline
\end{tabular}

peaks expected for the functional group. For example, if we use the presence of an $\mathrm{O}-\mathrm{H}$ peak to conclude "alcohol", we expect to find (and should look for) the presence of a $\mathrm{C}-\mathrm{O}$ peak to confirm the conclusion. Likewise, the presence of a $\mathrm{C}-\mathrm{H} \mathrm{sp}{ }^{2}$ peak, must mean that the spectrum contains a $\mathrm{C}=\mathrm{C}$ peak. Whenever possible, base our conclusion on all of the relevant data in the spectrum. Do not "cherry pick" your results.

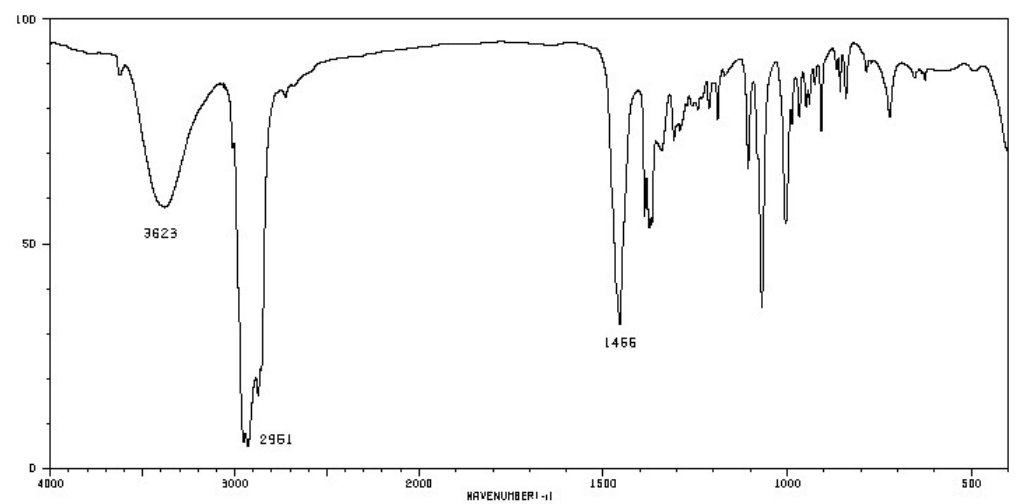

Figure 5.1. IR spectrum of alcohol starting material.

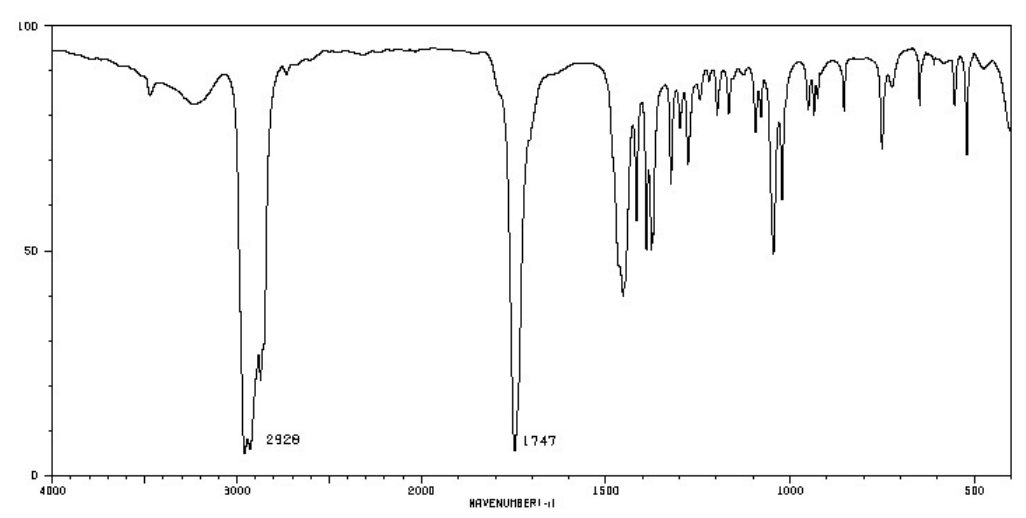

Figure 5.2. IR spectrum of stock sample of product ketone.
In general, it is a good idea to obtain an IR -spectrum of the starting material as well as an IR- spectrum of the product. This allows for a direct comparison of the two spectra and makes it easier to establish that the starting material is no longer present, and to find new peaks that might correspond to the product.

The spectra shown below come from an experiment in which a student tried to oxidize a secondary alcohol to a ketone. The top spectrum belongs to the starting material (alcohol), while the middle spectrum came from an authentic sample of the product. The bottom spectrum was obtained from a sample of the student's product.

The student's spectrum (bottom) contains a $\mathrm{C}=\mathrm{O}$ peak at $1747 \mathrm{~cm}^{-1}$, but a comparison of this spectrum with the starting material (top) reveals an alternative interpretation. The starting material's spectrum has a clear and distinct $\mathrm{OH}$-peak (and the corresponding 


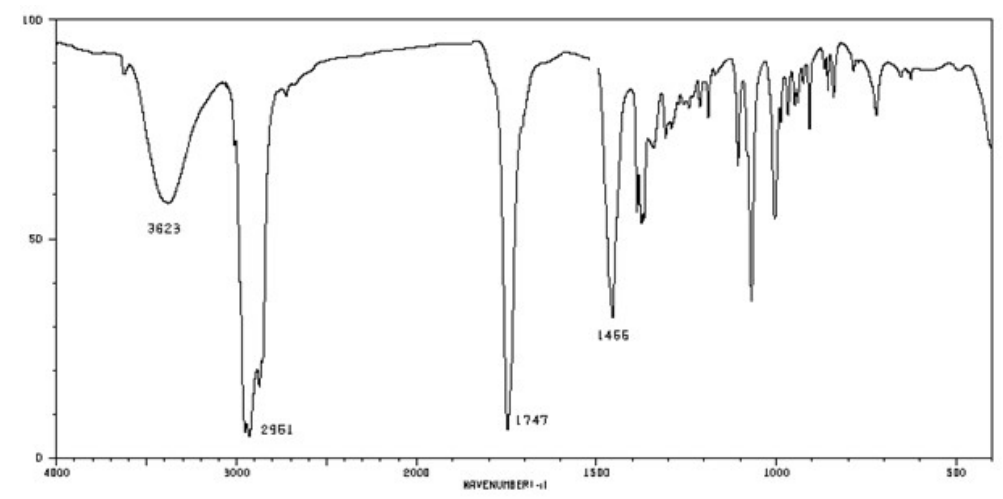

Figure 5.3. Student's sample of potential product.

$\mathrm{C}-\mathrm{O}$ peak). The spectrum from the stock sample of product, lacks the $\mathrm{OH}-$ peak, but a very characteristic $\mathrm{C}=\mathrm{O}$ peak is readily observed. The IR-spectrum of the student's product also indicates the presence of the desired ketone (strong $\mathrm{C}=\mathrm{O}$ peak), but it also reveals the same $\mathrm{O}-\mathrm{H}$ peak found in the starting material's spectrum.

One logical conclusion from this analysis is that the student's oxidation has not gone to completion or that the purification has not been satisfactory.

This is a microcosm of the entirety of IR -analysis: looking for the appearance of disappearance of specific peaks to answer the question of whether a reaction has worked or not.

\subsection{MELTING POINT ANALYSIS: IDENTITY AND PURITY}

The melting point of a compound is useful in two ways: it says something about the identity of a compound, and something about the purity of a compound. The presence of impurities will influence the melting point of a compound, leading to wider and depressed melting point ranges. Because even small deviations in melting point ranges is meaningful, it is worth mentioning that a control should be made when running the melting point of an isolated compound, against a stock sample of the same compound. In general, a deviation of more than $1 \mathrm{oC}$ in obtained melting point usually means the material is not of acceptable purity. Because the margin is relatively small, it is always worth running your sample with an authentic sample of the compound in the same melting point apparatus. If there are even small deviations in the apparatus, you will find that the authentic sample's melting point is also deviating from the literature value.

Melting points are usually not the only analytical data obtained for a sample; routinely IR-spectroscopy is also performed. Any abnormality in either data set should reflect in the other data source. For example, an IR spectrum corresponding to a product with a depressed melting point will often show peaks that are not expected for a pure sample. It is worth noting that this is not necessarily the case, as the impurities must also

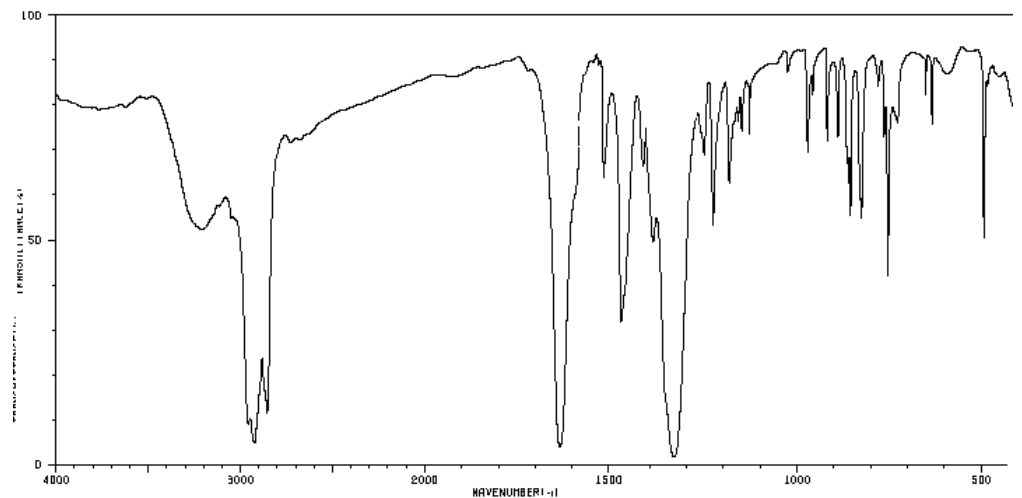

Figure 5.4. An IR spectrum obtained by a student. produce strong peaks in non-crowded areas of the IR-spectrum to be detectable.

Let us look at a specific example. A student recrystallized a sample of 2-naphthol from ethyl acetate. The published literature on 2-naphthol lists its melting point as $121-123{ }^{\circ} \mathrm{C}$, but the student's sample melted from 115$121{ }^{\circ} \mathrm{C}$, a range that was much broader and much lower than the literature one. 
These data are a clear indication that the product is not pure, but it always is preferable to be able to identify the source of the impurity as opposed to simply stating that there were impurities present. The key to this puzzle is found in the student's IR -spectrum.

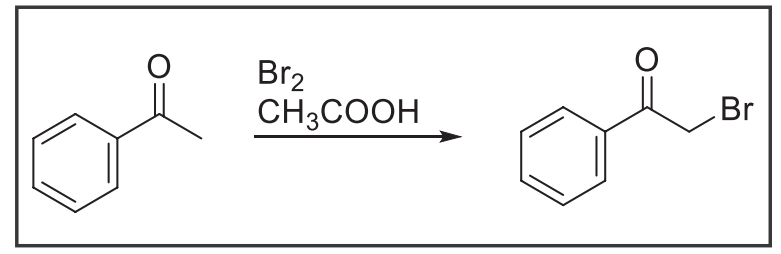

You will notice a clear indication of an $\mathrm{OH}$-bond (around $3200 \mathrm{~cm}^{-1}$ ) that is likely due to 2-naphthol, but

Scheme 1. Bromination of acetophenone.

you will also notice something else: a clear $\mathrm{C}=\mathrm{O}$ peak. It is very likely that this is trace ethyl acetate left over from the recrystallization, which indicates that the sample was not allowed to air-dry sufficiently.

A powerful observation is found when the two analyses are combined and viewed together.

\subsection{TLC: IDENTITY AND PURITY}

TLC can be a very useful resource to obtain quick and usefyul data about purity and identity. Unlike melting point analysis, where measurements can be compared to literature values, control TLC - experiments are essential to support the presence of compounds of interest. TLC has an advantage over melting point analysis, however, in that it can be applied to both liquids and solids.

Let us say that a student brominated acetophenone using bromine (scheme 1). Because the product contains a $\mathrm{C}$ - $\mathrm{Br}$ bond, IR -spectroscopy is not very useful (the peak assosicated with a $\mathrm{C}-\mathrm{Br}$ lies in a region of the spectrum that is hard to observe). Instead,

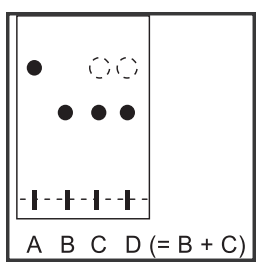

Figure 5.5. The TLC plate obtained by the student. the student performed a TLC analysis.

In order to get conclusive data from the TLC analysis, the student made up a TLC - plate with four samples. Spot $\mathrm{A}$ is the starting material acetophenone, spot $\mathrm{B}$ is a stock solution of the product, spot $\mathrm{C}$ is the product obtained by the student, and spot $\mathrm{D}$ is a co-spot of two solutions, the stock solution of product (spot B) and the student's product (spot C).

The TLC -analysis gives us important information about the experiment. The first result is the student's sample, $\mathrm{C}$, which appears to be two compounds. One of those (the darker spot) agrees with authentic product (B), but the other (the lighter spot) agrees with the starting material (A). This suggests that the student's product is contaminated with unreacted starting material. Spot D supports this interpretation: samples B and $\mathrm{C}$ produce a single dark spot in $\mathrm{D}$. 


\section{INTRODUCTION}

You prepared for the lab, finished the experiment, and now you have to compile a report. This writing assignment can in many ways be equally as challenging as the actual experiment itself. In this chapter, we will cover the best way to write a sound, well-written and scientifically coherent report.

\subsection{THE BASICS}

Different lab courses ${ }^{14}$ will have different report requirements, but the organic chemistry reports at PSU are based on the framework followed in most scientific research articles. These are typically divided into three parts:

1. The why: gives the information necessary to contextualize the experiment, states the goal of the experiment.

2. The how: gives information about how the experiment was performed

3. The what: provides relevant data and interpretation as it relates the goal of the experiment

Below is a table showing the different pieces that need to be included in your lab reports.

Table 1. Key goals of a report

\begin{tabular}{llll}
\hline$\#$ & Section & Name & Key goals of section \\
\hline 1 & Why & Title & A short title of the experiment. \\
2 & Why & Purpose of & The main goal and purpose of the experiment. \\
3 & How & Reaction scheme & The reaction scheme for the transformation attempted. \\
4 & How & Procedure & A description of the procedure is provided so that a professional can reproduce your experiment. \\
5 & What & Data & The key data obtained are presented, usually in tabulated form. \\
6 & What & Discussion & The results and findings from \#5 are discussed, and the outcome of the experiment as it pertains to \#2 is explained \\
7 & What & Conclusion & A conclusion is stated, based on the results. \\
8 & What & References & Relevant references are provided. \\
\hline
\end{tabular}

14 Different scientific disciplines, and even sub-disciplines, have different reporting requirements, different ways of presenting information, and different ways of using technical language. 
As the table shows, each section serves a distinct purpose and function. We will now look at each section in detail, and focus on the general goals of each, followed by things to watch out for, and common mistakes. The section numbers \#1-8 follow the sections used in the report forms at PSU.

\subsection{THE WHY: THE REASONS FOR DOING THE EXPERIMENT.}

\section{Section 1: Title}

A single sentence that fully captures the main goal of the experiment.

\section{Section 2: The Purpose of the Experiment}

(1) General: This section should be a brief statement about the ultimate goal of the experiment. Thisaection should never be longer than two sentences, and in many instances kept to only one sentence. Common goals are the identification of a compound, the synthesis of a compound, or the purification of a mixture. Techniques (TLC analysis, IR spectroscopy, extractions) should be mentioned only when these were the means to achieve the goal, and not a goal, and not the goal in themselves.

(2) Things to watch out for: The purpose must be result oriented, which means that general tasks like collecting data, performing calculations, getting results, and learning techniques, are inappropriate. Although learning to do new things is a top priority, it is never the purpose.

\section{(3) Some examples:}

Good: The purpose of the experiment was to synthesize benzoic acid through an oxidation reaction using bleach

Good: The purpose was to identify an unknown compound using TLC-analysis and melting point analyses.

Bad: The purpose of the experiment was to collect data and learn about recrystallization.

Bad: The purpose was to perform a four step synthesis that involved acetylation, hydrolysis, condensation and reduction, and use several new lab techniques such as extraction and sublimation. The student should learn about IR spectroscopy too, and analyze the data obtained.

\subsection{THE HOW: HOW WAS THE EXPERIMENT PERFORMED?}

\section{Section 3: Relevant Structures or Reaction scheme}

(1) General: Depending on the experiment, you should either supply structural formulas of the compounds you investigated, or provide a reaction scheme for a synthesis. Structural formulas should incorporate standard bond angles and can be hand-drawn. Usually the required structures are the compounds you investigated and listed in the purpose, or the reactants and the products you obtained through a chemical synthesis. Solvents are typically not required, nor are drying agents or other work-up compounds.

(2) Things to watch out for: Make sure that you provide structural, and not molecular formulae An example of the latter would be $\mathrm{C}_{6} \mathrm{H}_{12} \mathrm{O}_{6}$ for glucose. Molecular formulas have their place in organic chemistry, but are relatively useless for your report, as they do not provide any clarity in which bonds have been formed or broken. Take care to draw your formulae thatobey standard drawing conventions, i.e. with correct bond-angles, and/or correct atom hybridization. If you are drawing a reaction, draw molecules in the correct order: reactants on the left side of the reaction arrow, products on the right. Reagents, catalysts, solvents, and other important reaction conditions are drawn above and below the arrow.

\section{Section 4: Procedure}

(1) General: The procedure should encompass the core of the actions performed in the experimentation. It should contain the essential information as well as your exact measurements. The procedure must be written 
in past tense, and other verb forms such as present tense, or imperative form, must be avoided. You should assume that the reader has an extensive chemistry background, and is reading your procedure in order to learn how to repeat the experiment (note: the procedural format used in the lab manual is far too detailed for this reader). A challenging part of the procedure is to omit unnecessary details and focus on the necessities, see below for specific examples.

(2) Things to watch out for: Do not explain the reason for why anything is done in the procedure. That means that you might have added an acid to precipitate a product, but the reason for this action, is not appropriate. Furthermore, many actions that a chemist performs as part of an experiment should not be mentioned in the procedure. An example: before I can swirl a round bottom flask I must loosen the clamp, but the clamp adjustment should not be in the procedure because it follows logically. Likewise, I close the stop-cock on my separatory funnel before I pour materials into the funnel, but there is no need to mention this, or any of the other details on how I handled the funnel. A trained chemist will know how to handle a separatory funnel during a liquid-liquid extraction.

\section{(3) Some examples:}

Bad (explanations): $\mathrm{Na}_{2} \mathrm{SO}_{4}$ was added because it is a drying agent and therefore removes water from the crude reaction mixture.

Bad (wordy, imprecise): The distillation apparatus was assembled the way we have learned before and the reaction mixture was heated on a sand bath on a jack and the sand bath was set to approximately $350^{\circ} \mathrm{C}$. When I heated the mixture, it turned milky white, like a beautiful pearl that had been ground up and it smelled gross. I got a fraction boiling at $95-98^{\circ} \mathrm{C}$ that I think was the product but I'm not sure.

Good: Acetic acid $(1 \mathrm{~mL})$ and ethanol $(1 \mathrm{~mL})$ were mixed in a round bottom flask and heated to reflux.

Good: The reaction mixture was gravity filtered and the solvent removed using rotary evaporation.

\subsection{THE WHAT: WHAT WERE THE MAJOR FINDINGS OF THE EXPERIMENT?}

\section{Section 5. Data}

(1) General: Data that you collect must be presented clearly. In almost all cases, a tabulated form is required. The data that you should present is intimately tied to the purpose of the experiment. If the purpose is to synthesize a compound, the data are related to that purpose, i.e. they indicate whether or not the synthesis was successful.

Tables should be numbered and have captions. Units should be given in the title cells, in parentheses or brackets.

Most likely, the data will be analytical and spectroscopic data. Examples are:

- TLC-analysis (spots, distance travelled, $\mathrm{R}_{\mathrm{f}}$-values),

- Melting point data (melting point ranges, literature values)

- IR-spectroscopy data (functional groups, wavenumber, literature values)

- Synthesis data (only appropriate when a chemical synthesis has been performed): masses, volumes (if applicable), densities (if applicable), moles, yields

- NMR data (assignment, shift values, integrals)

In each table, the most important data should be presented. The task is to filter out the important data points. For example, when providing a table with IR data, you can readily find many peaks, but most of them are irrelevant to the purpose of an experiment or do not provide any information about the success of the experiment 
A key task is therefore to select and present meaningful data, and not just dump all your data into the report.

If you have acquired an IR (or NMR) spectrum, a copy of the spectrum should be included in this section.

(2) Things to watch out for: Two common examples of irrelevant data include mass of glassware and mass of filter paper. Appearance, consistency of a product and other physical properties of a similar nature, are also inappropriate. Make sure that the tables are formatted correctly.

\section{(3) Some examples:}

Good Table:

Table 1. Synthesis table

\begin{tabular}{lllll}
\hline Compound & Mw, [g/mol], & $\mathbf{m},[\mathrm{g}]$, & $\mathbf{n , ~ [ m m o l ] ,}$ & Yield, [\%], \\
\hline Benzaldehyde & 182 & 0.21 & 1.2 & - \\
$\mathrm{NaBH}_{4}$ & 38 & 0.10 & 2.6 & - \\
1,1-diphenylmethanol & 184 & 0.19 & 1.0 & 83 \\
\hline
\end{tabular}

Good Table:

Table 2. IR data

\begin{tabular}{lll}
\hline Bond & $\begin{array}{l}\text { Observed value } \\
{\left[\mathbf{c m}^{-1}\right]}\end{array}$ & $\begin{array}{l}\text { Literature value } \\
{\left[\mathbf{c m}^{-1}\right]}\end{array}$ \\
\hline $\mathrm{O}-\mathrm{H}$ & 3550 & $3200-3600$ \\
$\mathrm{C}-\mathrm{H}\left(\mathrm{sp}^{2}\right)$ & 3010 & $3090-3005$ \\
$\mathrm{C}-\mathrm{H}\left(\mathrm{sp}^{3}\right)$ & 2985 & $2985-2800$ \\
\hline
\end{tabular}

\section{SECTION 6. INTERPRET THE OBTAINED RESULTS (OR WHAT DID YOUR DATA MEAN?)}

(1) General: In many cases, interpretation of data is the most challenging component of any report. The key feature of an interpretation is that it says something about the data that relates to the purpose of that experiment.

There are two important scenarios: If the purpose of the experiment is to identify an unknown sample, then the interpretation extract information from the data that says something about the identity of the unknown. If the purpose of the experiment is to synthesize a compound, the interpretation must extract information that describes the identity and purity of the target compound.

The natural tendency is to write interpretations that are far too long. A good interpretation is exact, precise, and addresses the purpose of the experiment.

Such information can be:

1. TLC (says something about purity and identity)

2. Melting point (says something about purity and identity, especially when compared to a known melting point)

3. IR data (says something about which functional groups contained in the sample, which might indicate the presence or lack of the suspected product)

4. Yield (says something about the success of the reaction)

5. Boiling point (says something about the identity of the compound) 
Your data may not always support the expected outcome of an experiment. For example, your data may indicate that no reaction took place, or a product was very impure, and so on. These types of interpretations are appropriate as long as they are based on actual observations.

An example of a data interpretation that does not support the expected outcome is when you call attention to an $\mathrm{OH}$ peak in the IR spectrum that is not consistent with the structure of the desired product. That should be addressed, and a likely scenario to explain the presence of that peak should be provided.

(2) Things to watch out for: Unfocused or off-topic interpretations. Examples: interpretations that discuss what you learned, experiences you had or comments about your success as a lab student. None of these examples relates to the purpose of the experiment, and none of them deal with the objective data you collected. The interpretation should not deal with unimportant or irrelevant observations.

For example, if you say that the IR spectrum did not show the correct peaks because you collected the spectrum in a wrong way, that becomes a meaningless statement (how is it possible to collect a spectrum wrong, how do you know it is wrong, and why did you not address that issue if you knew about it?).

Here are some other examples with speculative conclusions that should be avoided.

- I weighed something wrong, or the scales were wrong

- The yield was low because I transferred the sample between vials

- The glassware used has a high degree of uncertainty

Furthermore, any error must have a direction and observations cannot contradict themselves.

If we come back to the example above with the $\mathrm{OH}$ peak that was found in your IR, that should not be there, surely that must mean that your yield also cannot be correct. And what about your melting point? That must also be off, or broader than it should be.

\section{(3) Some examples:}

Bad (imprecise, does not refer to specific data, no clear connection to the purpose of the experiment): The yield was higher than I wanted because of impurities and I weighed the sample wrong. The IR does not show what I should have because of something happening during the collection of the spectrum that should not have happened. The cylinder I used to measure was wet so that led to not getting correct data.

$\mathrm{Bad}$ (does not refer to any specific data, does not connect data to the purpose of the experiment, irrelevant): IR spectroscopy is used to saying something about which functional groups are found in an organic molecule. It can be used to find bonds that correspond to different functional groups. In my molecule, I have many bonds, and that can be seen in the spectrum. Furthermore, I have some strong peaks, and some weak ones, which is to be expected.

Good (Discusses specific data, interprets these data to support specific conclusion that relate to the purpose of the experiment): The yield was higher than expected (104\%) due to alcohol contaminants in the isolated product. This is evident from the IR-spectrum of the sample $\left(\mathrm{O}-\mathrm{H}\right.$ stretch at $\left.3551 \mathrm{~cm}^{-1}\right)$, which is a bond not present in the product. The melting point mirrors this $\left(74.1^{\circ} \mathrm{C}-84.9^{\circ} \mathrm{C}\right)$, as it is broader than expected $\left(81.5-85.0^{\circ} \mathrm{C}\right)^{1}$.

1. Correct citation to a literature source for the melting point. 
SECTION 7. CONCLUSIONS (OR WHAT WAS THE PRIMARY RESULT OF YOUR EXPERIMENT)

(1) General: The conclusion will be the final statement based on the success (or lack thereof) in the experiment. It will pertain to the purpose of the experiment. It will draw on the interpretation under \#6, and lead you to say something logical about the success of the experiment as a whole.

Some points that often are relevant are:

- Did you synthesize the suspected product (why, why not?)

- What was the purity of the compound isolated, what was the identity of the compound?

(2) Things to watch out for: Conclusions that are not anchored in the purpose of the experiment should be avoided. If you have recrystallized a product, concluding about how much you liked or did not like the lab, is not appropriate. Subjective opinions and learning outcomes, although important to us, are not things that should be placed in the conclusion. Also, avoid using statements about what you would have done differently.

\section{(3) Some examples:}

Bad (irrelevant): I really liked learning about melting point analysis and I can see that it will be valuable for me in the future, because I am becoming a melting point expert.

Good (says something about the success of the experiment and its purpose): Benzoic acid was prepared in a moderate yield. The product isolated is likely benzoic acid, as it shows a similar IR spectrum that contains the key functional groups. 